\title{
Caracterización Sismotectónica de México
}

\section{Seismotectonic characterization of Mexico}

\author{
Mario Octavio Cotilla-Rodríguez ${ }^{1}$ \\ Diego Córdoba-Barba \\ Universidad Complutense de Madrid, España \\ Francisco Javier Núnez-Cornü ${ }^{3}$ \\ Universidad de Guadalajara, México
}

\begin{abstract}
Resumen
México es una Región Sismotectónica activa, mayoritariamente en la placa continental de Norteamérica y tiene los 2 tipos de sismicidad (entreplacas y de interior de placa). La estructura jerárquica contiene 3 Provincias Sismotectónicas (Norte-Occidental, Occidental y Centro-Oriental), en ellas hay 11 Unidades Sismotectónicas y en éstas se localizan las zonas sismogenéticas. Estas últimas están segmentadas. La Provincia Occidental es la de más nivel y donde se encuentra el contacto de las placas convergentes.
\end{abstract}

Palabras clave: México, peligrosidad sísmica, sismotectónica, zonas activas.

\begin{abstract}
Mexico is an active seismotectonic region mainly sitting on the continental plate of North America and has two types of seismicity: interplate and interior plate. The hierarchical structure embraces 3 seismotectonic provinces (north-western, western and central-eastern), comprised of 11 seismotectonic units wherein seismogenetic zones are located. The latter are segmented. The western province shows the highest level of seismicity and is the area where convergent plates make contact.
\end{abstract}

Keywords: Mexico; seismic hazard; seismotectonics; active zones

1 Universidad Complutense de Madrid. Facultad de Ciencias Físicas. Departamento de Física de la Tierra y Astrofísica. Ciudad Universitaria s/n. 28040, Madrid. Correo electrónico: macot@ucm.es

2 Universidad Complutense de Madrid. Facultad de Ciencias Físicas. Departamento de Física de la Tierra y Astrofísica. Ciudad Universitaria s/n. 28040, Madrid. Correo electrónico: dcordoba@fis.ucm.es

3 Centro de Sismología y Volcanología de Occidente, Centro Universitario de la Costa, Universidad de Guadalajara, México, Correo electrónico: pacornu77@gmail.com 


\section{Introducción}

México $[\mathrm{MX}]\left(\sim 1.964 .400 \mathrm{~km}^{2}\right)$ localizado en la placa de Norteamérica es una estructura neotectónica vinculada al Océano Pacífico [OP], Golfo de México [GM] y Mar Caribe (Figura 1). Las costas tienen 11.600 km (2,7 Occidental $>$ Oriental $)$. Hay 31 Estados $\left(\sim 122.10^{6}\right.$ habitantes $)$ y poblaciones con áreas turísticas (playas) del OP $\left(>10.10^{6}\right.$ habitantes/ $\sim 30.10^{6}$ turistas/ año) sujetos a tsunamis y terremotos (07.09.2017 M 8,2/ 102 muertos/ 900 heridos/ 2.500.000 afectados/ tsunami en Oaxaca [OX]; 19.09.2017 M 7,1/ Morelos/ 370 muertos/ 7.289 heridos). Otras áreas de costa del GM e interior con interés turístico-cultural son: 1) playas (Cancún, Tuxpan, Boca del Río, Rivera Maya); 2) parques temáticos (El Pinacote, Gran Desierto de Altar); 3) volcanes (Ceboruco, Popocatépetl). Todas tienen peligro sísmico y más cantidad de personas que en el OP. El Proyecto TSUJAL realiza el trabajo para dar las bases de una regionalización sismotectónica [ST] y confeccionar, en una segunda etapa, un mapa sismotectónico [MS].

\section{Sismotectónica}

Un MS es un material sismológico especial, dinámico, no probabilístico, ni ecléctico, que debe ser claro, conciso y objetivo. Documento fundamental para estudios de peligrosidad sísmica, válido para un periodo determinado. África, Cuba, España, Francia, Italia, Japón, Rumania y Rusia los tienen. Criterios para su preparación están en Pavoni (1985) y Cotilla et al. (1991) destacando los datos sobre: $\underline{\mathbf{1}}$ neotectónica, 2) sismicidad

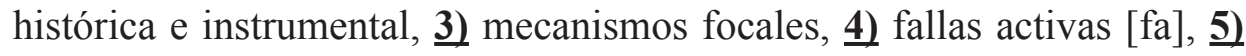
estructura de la corteza. NUREG-1451 (1992) y Regulatory Guide 1.165 (1997) recomiendan: 1) incluir fallas, 2) realizar una regionalización. Identifican y clasifican las fallas y áreas activas. Según especialistas norteamericanos ST es el estudio de terremotos-tectónica activa-fallas de una región. De otra parte, especialistas rusos consideran: 1) preparación del MS, 2) caracterización de zonas sísmicas potencialmente activas, $\underline{\mathbf{3}}$ ) elaboración de materiales de peligrosidad sísmica. Estudiar una fa es una etapa inicial. Ellas tienen actividad sísmica [AS], magnitud máxima [Mmáx] y periodo de recurrencia de los terremotos, que no siempre es posible de establecer (Trifonov y Machette, 1993). Están en un régimen tectónico regional, y Provincias Sismotectónicas [PS] y Unidades Sismotectónicas [US]. En MX hay catálogos: 1) Península de California [PC], 2) MX. 


\section{Figura 1. Esquema tectónico}

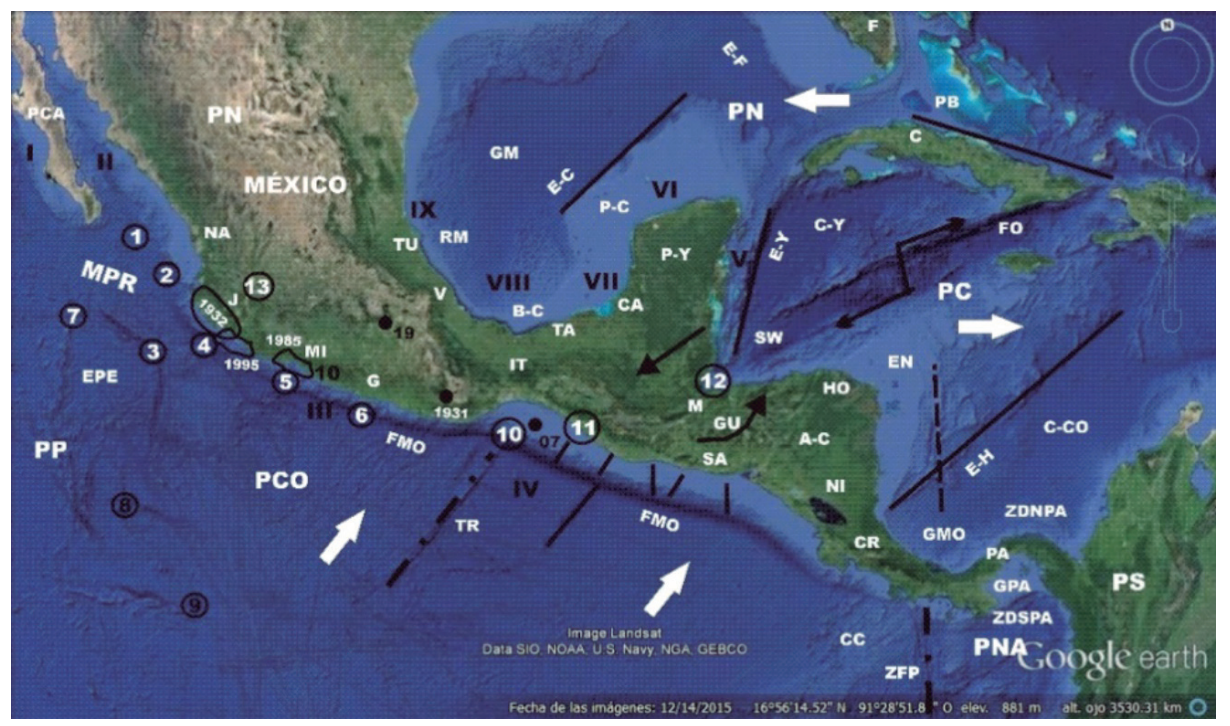

Aparecen: 1) Lugares (A-C = América Central, $\mathrm{B}-\mathrm{C}=$ Bahía de Campeche, $\mathrm{C}=\mathrm{Cuba}, \mathrm{CA}=\mathrm{Cam}$ peche, $\mathrm{CC}=$ Cresta de $\mathrm{Cocos}, \mathrm{CR}=$ Costa Rica, Cuenca $(\mathrm{C}-\mathrm{CO}=$ de Colombia, $\mathrm{CY}=$ de Yucatán), Escarpe $(\mathrm{E}-\mathrm{C}=$ de Campeche, $\mathrm{E}-\mathrm{H}=$ de Hess, E-F= Farallón, E-Y= de Yucatán), Elevado $(\mathrm{EN}=\mathrm{de}$ Nicaragua, EPE= del Pacífico Este), $F=$ Florida, Fosa $(F O=$ Oriente, $F M O=$ Mesoamericana $), G=$ Guerrero, Golfo (GM= de México, GMO=de Mosquitos, GPA=de Panamá), GU= Guatemala, $\mathrm{HO}=$ Honduras, IT= Istmo de Tehuantepec, $\mathrm{J}=$ Jalisco, $\mathrm{M}=$ Motagua, $\mathrm{MI}=$ Michoacán, NA= Nayarit, NI= Nicaragua, $\mathrm{PA}=$ Panamá, Plataforma $(\mathrm{PB}=$ de Bahamas, $\mathrm{P}-\mathrm{C}=$ de Campeche), Península $(\mathrm{PCA}=\mathrm{de}$ California, $\mathrm{P}-\mathrm{Y}=$ de Yucatán), $\mathrm{RM}=$ Ridge de México, $\mathrm{SA}=$ El Salvador, $\mathrm{SW}=$ Falla de $\mathrm{Swan}, \mathrm{TA}=$ Tabasco, TU= Tuxpan, V= Veracruz, Zonas de Deformación de Panamá (Norte= ZDNPA, Sur= ZDSPA), (líneas cortas y puntos negros: (TR= Tehuantepec Ridge, ZFP= Zona de Fracturas de Panamá)); 2) círculos negros (epicentros de terremotos: 07=07.09.2017 M 8,2, 19=19.09.2017 M $7,1,1931=15.01 .1931$ M 8,0; 3) circulo (negro) con número (blanco)= intersección principal de fallas; 4) Elipses (áreas de rupturas estimadas de los terremotos: 03.06.1932 M 8,2, 19.09.1985 M 8,1, 09.10.1995 M 8,0); 5) Microplacas (MPR=Rivera); 6) Segmentos de costa (I-IX); 7) Placas $(\mathrm{PC}=$ Caribe, $\mathrm{PCO}=$ Cocos, $\mathrm{PN}=$ Norteamérica, $\mathrm{PNA}=$ Nazca, $\mathrm{PP}=$ Pacífico, $\mathrm{PS}=\mathrm{Suramérica}) ; 8)$ Sentido de movimiento (Placas $=$ flechas gruesas blancas, $\mathrm{y}$ Bloques $=$ flechas negras finas).

Zúñiga et al. (1997) dijeron: “...Basándose en los datos de los catálogos instrumental e histórico, el territorio de México se subdividió, de manera global, en Unidades Sismotectónicas con el propósito de posteriormente definir dentro de estas Zonas Sismogenéticas para ser utilizadas en las estimaciones del Riesgo ...". Los resultados de Montero et al. (1997) y Zúñiga et al. $(1997,2017)$ siguen la teoría de los norteamericanos, cubren el periodo 1980-2017, y los mapas ST de los segundos autores 
difieren ligeramente (19 y 18 regiones, respectivamente) y sitúan las zonas más activas en el contacto de placas. A partir de sus observaciones: 1) “[...] es de ningún modo única [...]”, 2) “[...]pueden realizarse particiones más finas y precisas [...]" se realiza este trabajo.

La confección de un MS utiliza la experiencia documentada de otros especialistas. Es conocido que una fa: $\underline{1}$ ) está sujeta a procesos de acumulación y liberación de energía, que no siempre se ajustan a ciclos perfectos; 2) que esos procesos son independientes; 3) que los límites político-administrativos limitan el alcance de las investigaciones sismotectónicas. Guzmán Speziale y Gómez (2006) demuestran diferencias de AS del Occidente de MX con los pares de placas Pacífico-Norteamérica, Cocos-Norteamérica y Cocos-Caribe. Lyon Caen et al. (2006) determinan la diferencial cinemática de las placas Cocos-Norteamérica-Caribe. Guendel y Protti (1998) analizan la ST de América Central [AC]. DeShon et al. (2003) determinan la sismogenésis de la zona del OP (Honduras-Costa Rica). MX tiene heterogeneidad (microplacas, bloques y zonas de deformación) y los trabajos de tectónica y morfotectónica, a diferentes escalas para áreas y regiones diversas, y constata la segmentación de estructuras con intersecciones, que en el campo geofísico están comprobadas; por lo que una fa no es extensa, regular, homogénea, ni completamente nacional. La segmentación de una fa tiene su antecedente en Wheeler y Krystinik (1827). Suarez y Comte (1992) comparan la ST de MX y Chile.

\section{Apuntes sobre Geología-Tectónica}

Resultados de geología, geomorfología y tectónica (Dañobeitia et al., 2016; DeMets et al., 2007, Frez y González, 1991; Guzmán Speziale y Meneses Rocha, 2000; Michaud et al., 2000; Núñez Cornú et al., 2016) permiten decir que los países del Pacífico americano tienen relación las placas Caribe-Norteamérica-Cocos-Nazca y la microplaca Rivera [MPR]. Ellos son parte del "Cinturón de Fuego del Pacífico" y tienen diferencias en morfología, hipsometría, velocidad de movimiento, nivel de AS y volcánica, y tipo de fuente sismogenética (de interior y de entre placas). En el mapa de MX se aprecian la geometría, configuración y elementos principales y destaca el contacto geodinámico de las placas Cocos-Caribe y la forma arqueada (al S de Chiapas-Panamá). La fosa Mesoamericana [FM] es una estructura, deprimida y relativamente estrecha, con sismicidad; localizada 
en la costa Nayarit-Puerto Vallarta [PV]-S de Chile (Figuras 2-3). Ese entorno cuasi-lineal tiene montañas, fosas y volcanes, y se aprecia la diversidad de las zonas de subducción con sus perfiles de Wadati-Benioff [WB], asociadas con los terremotos (Tabla 1) tsunamis y volcanes. La zona de subducción es una franja inmediata a la costa y parte de la FM está definida por la geometría batimétrica, la localización de las cadenas volcánicas y la AS. La zona de WB destaca por el buzamiento $\left(50^{\circ}-70^{\circ}\right)$ e hipocentros $>200 \mathrm{~km}$, y para Nayarit-Chiapas se diferencia por: 1) edad estimada $\left(10^{6}\right.$ años) de 8 a 15, 2) forma del eje central (cóncavo-lineal-cóncavo).

Figura 2. Región de Chiapas-Guatemala

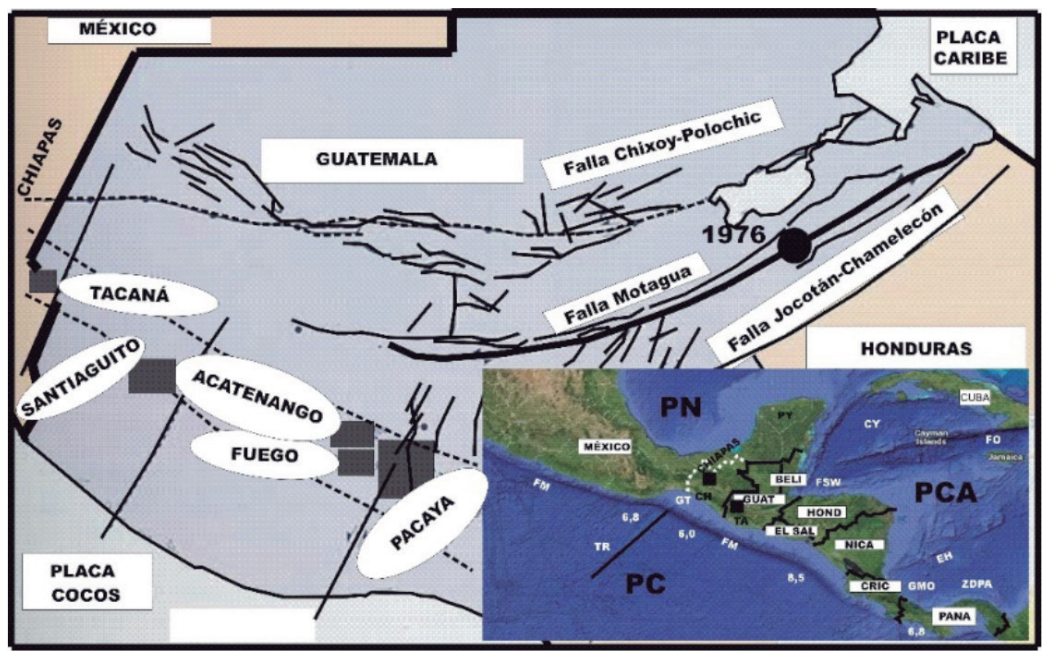

Aparecen: 1) Principales fallas y fracturas (líneas negras); 2) Volcanes activos (rectángulos grises); 3) Epicentro del 04.02.1976 M 7,6; y 4) Recuadro: A) países al S y E de México (BELI= Belize, CRIC $=$ Costa Rica, EL SAL $=$ El Salvador, GUAT $=$ Guatemala, HOND $=$ Honduras, NICA $=$ Nicaragua, $\mathrm{PANA}=$ Panamá); $\mathrm{B})$ Placas $(\mathrm{PC}=\mathrm{Cocos}, \mathrm{PCA}=$ Caribe, $\mathrm{PN}=$ Norteamérica); $\mathrm{C})$ Volcanes $(\mathrm{CH}=$ Chichón (Chiapas-México), $\mathrm{TA}=$ Tacaná (Guatemala), con cuadrados negros); D) velocidad relativa (cm/año); E) Lugares: $\mathrm{CY}=$ Cuenca de Yucatán, EH= Escarpe de Hess, Fosa (FM= Mesoamericana, $\mathrm{FO}=$ Oriente), $\mathrm{SW}=$ Falla Swan, Golfo $(\mathrm{GMO}=$ de los Mosquitos, $\mathrm{GT}=$ de Tehuantepec $)$, PY= Península de Yucatán, TR= Tehuantepec Ridge, ZDPA= Zona de deformación de Panamá).

La placa Pacífico abarca la mayor parte del OP y en el E limita con las placas de Norteamérica y de Suramérica, y se definen microplacas (Juan de Fuca, Gorda y Explorador asociadas con la fa San Andrés). Se 
configura la cadena volcánica Cascadia, y más al S, de la PC, aparecen la MPR, las placas Cocos $\left(3.10^{6} \mathrm{~km}^{2}\right)$ y Nazca; que son restos de la placa Farallón (23.10 años). Las 2 primeras subducen la norteamericana y vinculan con la Cadena Volcánica Trans-mejicana (Eje Neovolcánico [ENV]) (Figura 3). La MPR se separó de la placa Cocos hace 5-10.106 años y se desplaza con velocidad de 2,5 cm/año y la placa Cocos con 5 y $8 \mathrm{~cm} /$ año en Manzanillo y Tehuantepec, respectivamente. En la zona marina S del Golfo-PC-NO de PV hay un cambio estructural con 3 sistemas de fallas del tipo: 1) transcurrente, 2) inverso (subducción asociada a la FM), 3) normal (transversal a los anteriores y que se corresponde con el graben de Vallarta). Hay 3 zonas relacionadas con la intersección de grandes estructuras (Puntos Triples de las placas): 1) Pacífico-Norteamérica (Cerro PrietoLaguna Salada-Golfo de California), 2) Norteamérica-Cocos-Rivera (Baja California-PV), 3) Caribe-Cocos-Norteamérica (Chiapas-Guatemala) (Figuras 1-4). La primera intersección (Punta Borrascosa) es la bifurcación de la fa San Andrés en un sistema cuasi paralelo (Cerro Prieto, Amado y Wagner) denominado Fracturas del Golfo. Otra intersección en Colima-Laguna de Chapala-Tepic-Zacoalco es de menor categoría y actividad, y configura con 3 graben (NO-SE (Tepic-Zacoalco), E-O (Chapala), N-S (Colima)). El primero y tercero tienen volcanes (Sanganguey, Ceboruco, Colima); y todos se vinculan con fallas de tipo normal, la mayoría inactivas. En la laguna de Chapala se determina un tensor ENE.

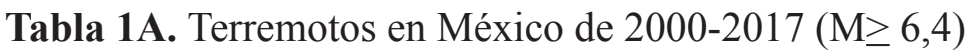

\begin{tabular}{|c|c|c|c|c|c|}
\hline Fecha & $\mathbf{M} / \mathbf{h}(\mathbf{k m})$ & Coordenadas (N / O) & Fecha & $\mathbf{M} / \mathbf{h}(\mathbf{k m})$ & Coordenadas (N / O) \\
\hline 2000.08 .09 & $7,0 / 9$ & $17,94 / 102,71$ & 2012.03 .20 & $7,4 / 16$ & $16,25 / 98,52$ \\
2001.05 .19 & $6,5 / 20$ & $18,27 / 105,72$ & .04 .11 & $6,4 / 16$ & $17,90 / 103,06$ \\
2003.01 .21 & $7,6 / 10$ & $18,22 / 104,60$ & .12 & $6,8 / 10$ & $28,78 / 113,43$ \\
2006.01 .04 & $6,7 / 10$ & $28,10 / 112,07$ & .11 .07 & $7,3 / 16$ & $14,08 / 93,32$ \\
2008.02 .12 & $6,6 / 90$ & $16,19 / 94,54$ & 2014.04 .18 & $7,2 / 10$ & $17,18 / 101,19$ \\
.09 .23 & $6,4 / 42$ & $17,16 / 105,16$ & .05 .08 & $6,4 / 17$ & $17,11 / 100,87$ \\
.10 .26 & $6,6 / 23$ & $13,87 / 92,50$ & .07 .07 & $6,9 / 60$ & $14,75 / 92,63$ \\
2009.08 .03 & $6,9 / 10$ & $28,48 / 112,24$ & .29 & $6,4 / 117$ & $17,70 / 95,63$ \\
2010.04 .04 & $7,2 / 10$ & $32,54 / 115,36$ & 2015.09 .15 & $6,7 / 10$ & $24,96 / 109,49$ \\
.10 .21 & $6,5 / 8$ & $24,62 / 109,43$ & .12 .17 & $6,6 / 90$ & $15,76 / 93,70$ \\
2011.04 .07 & $6,7 / 167$ & $17,20 / 94,34$ & 2017.09 .07 & $8,2 / 58$ & $14,85 / 94,11$ \\
.12 .10 & $6,5 / 58$ & $17,84 / 99.98$ & .19 & $7,1 / 57$ & $18,40 / 98,72$ \\
\hline
\end{tabular}


Mario Octavio Cotilla-Rodríguez - Diego Córdoba-Barba - Francisco Javier Núnez-Cornú Caracterización Sismotectónica de México

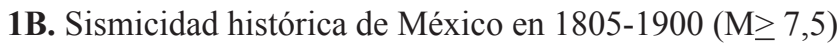

\begin{tabular}{|c|c|c|c|c|c|}
\hline M & Fecha & Coordenadas (N / O) & M & Fecha & Coordenadas (N / O) \\
\hline 7,5 & 1805.03 .25 & $18,90 / 103,89$ & 7,7 & 1818.05 .31 & $19,10 / 103,60$ \\
& 1845.03 .09 & $16,60 / 97,00$ & & 1837.11 .22 & $20,00 / 105,00$ \\
& 1858.06 .19 & $19,60 / 101,60$ & & 1854.05 .05 & $16,30 / 97,30$ \\
& 1875.02 .11 & $21,00 / 103,80$ & 7,9 & 1845.04 .07 & $16,60 / 99,20$ \\
& 1882.07 .19 & $17,70 / 98,20$ & & 1870.05 .11 & $15,80 / 96,70$ \\
7,6 & 1806.03 .25 & $20,22 / 103,41$ & & 1899.06 .24 & $17,10 / 100,50$ \\
& 1820.05 .04 & $17,20 / 99,60$ & & 1900.01 .20 & $20,00 / 105,00$ \\
\hline
\end{tabular}

1C. Sismicidad histórica más relevante por regiones (1568-1870)

\begin{tabular}{|c|c|c|c|c|c|}
\hline Fecha & Intensidad & Región & Fecha & Intensidad & Región \\
\hline 1568.12 .27 & X & Jalisco & 1787.03 .28 & IX & Guerrero-Oaxaca \\
1604.03 .03 & X & Oaxaca & 1697.02 .07 & IX & Guerrero \\
1682.03 .19 & VIII-IX & & 1754.09 .01 & XI & \\
1696.08 .23 & IX & & 1820.05 .04 & IX & \\
1727.03 .10 & IX & & 1845.04 .07 & X & \\
1787.04 .03 & IX & & 1711.08 .15 & IX & Puebla-Tlaxcala \\
1795.03 .23 & VIII-IX & & 1864.10 .03 & X & \\
1801.10 .05 & IX & & 1776.04 .21 & IX & Acapulco \\
1870.05 .11 & X & & 1858.06 .19 & X & Michoacán \\
1882.07 .19 & IX & & 1806.03 .25 & VII & Colima \\
\cline { 5 - 6 } & & & 1818.05 .31 & X & Jalisco-Colima \\
\hline
\end{tabular}

Se conoce que: 1) la fractura de Tehuantepec afecta la FM, divide la placa Cocos, y se extiende hacia Chiapas; 2) la zona de subducción aparece dividida, sucesiva y lateralmente, en segmentos de 100-300 km; 3) los principales rasgos morfotectónicos del talud interior de la FM se continúan al interior, como la falla Tecpan (en Guerrero), por más de $300 \mathrm{~km}$. En las inmediaciones de Manzanillo, está el graben El Gordo (SSO-NNE) asociado espacialmente con el graben de Colima (dirección S-N), donde hay volcanes. Además, hay AS (09.10.1995) incluidos tsunamis en Manzanillo. Todos esos elementos lineales están en la zona límite de placas [ZLP] del Pacífico. De Arnaiz Rodríguez y Garzón (2012) se interpretan los mapas de: 1) Anomalías de Bouguer Total, que tiene las grandes estructuras del entorno $100^{\circ}$ \% $50^{\circ} \mathrm{O}$ y $5^{\circ} / 30^{\circ} \mathrm{N}$; y la zona continental de América del Norte-MX-América del Sur con valores mínimos que se relacionan con la subducción, y en la 
placa Cocos un máximo de $279-340$ mGal. Se dice es joven; 2) Flujo de Calor de la Corteza, en el sector de MX, muestra la diferenciación entre la zona Norte-Central-Manzanillo y el ENV con respecto al S de la Cadena en OX-Chiapas-Yucatán; 3) el mapa del espesor continental de Norteamérica distingue una distribución transversal con incremento al E. Verma (1985) confirma que la cadena volcánica en la zona costera está asociada a la subducción. La placa que subduce lo hace con distinto ángulo, profundidad, actividad y edad en diferentes segmentos de la costa. El otro conjunto transversal (ENV), aunque su morfología y disposición puede vincularse a la subducción de la MPR. Este tiene 4 volcanes (altitud (m)/ año última actividad): Ceboruco (2.280/1875), Colima (3.880/2016), Popocatépetl (5.550/2016). Otros volcanes con peligro son: El Chichón (1.315/1982), Pico de Orizaba (3.690/1858), San Martín de Tuxla (1.650/1796), Tacaná (S de Chiapas 4.067/1986)). Una comparación entre MX y otros países está en la tabla 2.

Tabla 2. Datos de países del Pacífico americano al sur de México

\begin{tabular}{|c|c|c|c|}
\hline $\begin{array}{c}\text { País/ área }\left(\mathbf{k m}^{2}\right) / \\
\text { población }\end{array}$ & $\begin{array}{c}\text { Mmáx/ h }(\mathbf{k m}) / \\
\text { fallecidos/ volcanes/ tsunamis }\end{array}$ & $\begin{array}{c}\text { País/área }\left(\mathbf{k m}^{2}\right) / \\
\text { población }\end{array}$ & $\begin{array}{c}\text { Mmáx/ h }(\mathbf{k m}) / \\
\text { fallecidos/ volcanes/ } \\
\text { tsunamis }\end{array}$ \\
\hline $\begin{array}{c}\text { El Salvador/ } 21.10^{3} / \\
733.10^{4}\end{array}$ & $8,1 / 25 / 400 / 22 / 3$ & $\begin{array}{c}\text { Honduras/ } 112,5.10^{3} / \\
911,5.10^{4}\end{array}$ & $7,3 / 10 / 7 / 4 / 7$ \\
$\begin{array}{c}\text { Chile/ } 756.10^{3} / \\
17,6.10^{6}\end{array}$ & $9,6 / 35 / 1.655-2.000 / \sim 500 / 35$ & $\begin{array}{c}\text { Nicaragua/ } 62.10^{5} / \\
130,4.10^{3}\end{array}$ & $7,9 / 45 / 22.870 / 21 / 8$ \\
Costa Rica/ $51.10^{3} /$ & $7,6 / 10 / 125 / 17 / 15$ & $\begin{array}{c}\text { Panamá/ } 75,5.10^{3} / \\
4,1.10^{6}\end{array}$ & $7,9 /-/ 5 / 3 / 4$ \\
$4,9.10^{6}$ & $7,9 / 60 / 22.870 / 37 / 5$ & Perú/ $1,3.10^{6} / 32.10^{6}$ & $8,4 / 33 / 240 / \sim 400 / 123$ \\
\hline $\begin{array}{c}\text { Guatemala/ } 109.10^{3} / \\
17,4.10^{6}\end{array}$ & &
\end{tabular}

El límite del S (entre las placas de Norteamérica-Caribe, y la placa Cocos) está dado por un sistema de fallas cuasi paralelas (Chixoy-Polochic, Motagua, Jocotán-Chamelecón) localizadas en Guatemala. Tienen dirección aproximada E-O y son transcurrentes, pero su AS es distinta. La fa Motagua se asocia al terremoto del 04.02.1976 M 7,9/ h 5 km/ 22.870 muertos/ 76.504 heridos $/>1.10^{6}$ damnificados, y tiene relación al E con la falla Swan. La falla del Polochic no se relaciona con Motagua; su trazo es continuo hasta Chiapas y divide en 2. Authemayou et al. (2012) sostienen que es activa. La falla Jocotán no tiene AS reconocida, e identifica por 
segmentos como falla normal. Las medidas de GPS dan una velocidad de 1,7 $\mathrm{cm} /$ año entre las mencionadas placas, con disminución al OP. Authemayou et al. (2011) sostienen que la intersección de las placas es un punto triple activo, con un modelo "pull-up and zipper" (Polochic-Motagua). Cotilla et al. (1991) proponen un sistema bloques y fa, en la interacción de placas CaribeNorteamérica-Cocos; y consideran las fallas Motagua y Swan.

Figura 3. Principales placas
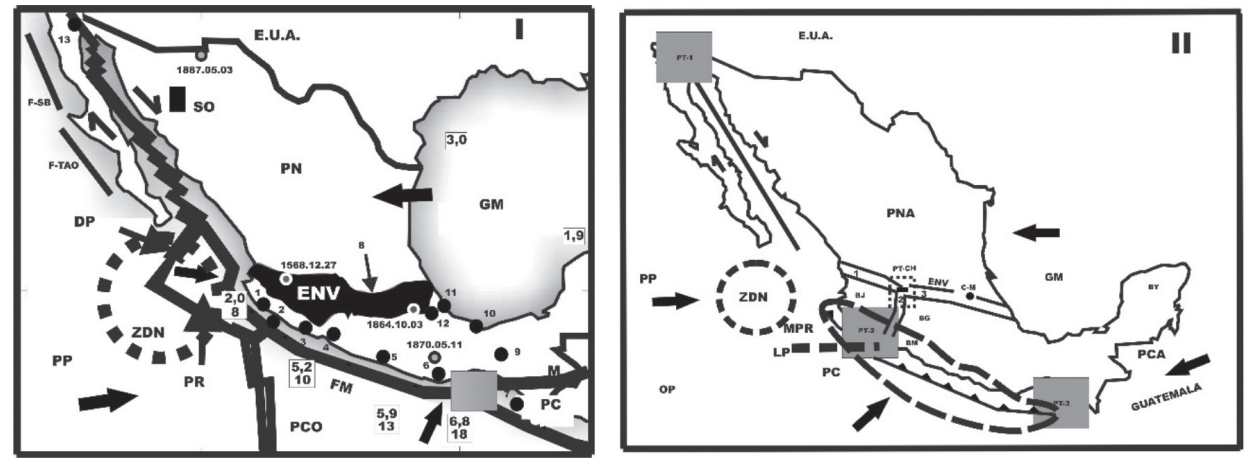

I: 1) $P$ lacas $(\mathrm{PC}=$ Caribe, $\mathrm{PCO}=\mathrm{Cocos}, \mathrm{PN}=$ Norteamérica, $\mathrm{PP}=$ Pacífico, y $\mathrm{PR}=$ Rivera $)$;

2) Elementos $(\mathrm{DP}=$ Dorsal del Pacífico, $\mathrm{ENV}=$ Eje Neovolcánico, E.U.A. $=$ Estados Unidos de Norteamérica, FM= Fosa Mesoamericana, Falla $(\mathrm{F}-\mathrm{SB}=$ de San Benito, F-TAO= ToscoAbreojos), $\mathrm{GM}=$ Golfo de México, $\mathrm{M}=$ Motagua, $\mathrm{SO}=$ Sonora (rectángulo negro), $\mathrm{ZDN}=$ Zona de deformación neotectónica (círculo de puntos negros); 3) Sentido de movimiento de las: (A) placas (flechas negras gruesas), B) fallas (flechas negras finas)); 4) Fallas y fracturas (líneas negras); 5) velocidad de movimiento ( $\mathrm{cm} / \mathrm{año})$, edad $\left(10^{6}\right.$ años) aparecen en un cuadrado; 6) Epicentros de terremotos: A) (circulo negro y número asociado [1=1995.10.09 M 8, 2= 2003.01.21 M 7,6, $3=1985.09 .19$ M 8,1, $4=1985.09 .217,6,5=2012.03 .20 \mathrm{M}$ $7,4,6=1999.09 .30$ M 7,4, 7=2017.09.07 M 8,2, $8=2017.09 .19$ M 7,0, $9=1902.09 .23 \mathrm{M}$ $7,8,10=1959.08 .26$ M 6,4, $11=1973.08 .28$ M 7,3, 12=1920.01.03 M 8,0, 13=2010.04.04 M 7,2]); B) 4 históricos con I= X grados (circulo gris y fecha asociada)); 7) Zona de intersección Chiapas-Guatemala (rectángulo gris), que incluye al volcán Tacaná. En ei entorno está el sismo del SO de Tapachuela, Chiapas (frontera Guatemala-México) del 07.07.2014. II: 1) Lugares (C-M= Ciudad de México, ENV= Eje Neovolcánico, E.U.A.= Estados Unidos de América, $\mathrm{GM}=$ Golfo de México, $\mathrm{LP}=$ Límite de placas, $\mathrm{OP}=$ Océano Pacífico); Placas $(\mathrm{PCO}=\mathrm{Cocos}$, $\mathrm{PCA}=$ Caribe, $\mathrm{PNA}=$ Norteamérica, $\mathrm{PP}=$ Pacífico), y MPR $=$ Microplaca Rivera; 2) Sentido de movimiento de las placas (flechas gruesas negras); 3) Zona de deformación neotectónica (ZDN: circulo con puntos); 4) Punto Triple (A) cuadrado gris (PT-1= Cerro Prieto-Laguna Salada (Punta Borrascosa), PT-2= California-Vallarta y PT-3= Chiapas-Guatemala), B) rectángulo de puntos con rectángulo negro en el centro (PT-CH= Chapala; 5) Graben ( $1=$ Tepic-Zacoalco, $2=$ Colima, $3=$ Chapala); 6) Zona epicentral significativa (elipse con línea discontinua); 7) Zona de subducción (línea negra con triángulos), 8) Fracturas y fallas del Golfo de California (con sentido de movimiento relativo). 
Al S de MX (Chiapas-Guatemala-Caribe) hay interrupción del relieve montañoso (en cuanto a niveles hipsométricos). El acoplamiento de las placas Caribe-Cocos es suave y la zona de WB es más superficial. En AC es mayor el buzamiento $\left(60^{\circ}-80^{\circ}\right.$ y dirección NE) con hipocentros de $200 \mathrm{~km}$. En la costa se identifican segmentos y diferencias en rumbo y buzamiento de los planos, que corresponden a mecanismos focales a distinta profundidad. En los primeros $10 \mathrm{~km}$ hay fallas de tipo normal, que a 15-50 $\mathrm{km}$ son inversas. Esto es un marco activo. La zonación se aviene con la morfología y disposición de los volcanes, el patrón de fa, las alineaciones transversales y ocurrencia de terremotos someros. Hay rupturas diversas de las placas de subducción, y la tectónica resulta compleja al E de la Cresta de Panamá, donde las Fracturas de Panamá. La ZLP de Cocos-Nazca tiene mecanismos de tipo transcurrente, y la segunda placa penetra con buzamiento suave y oblicuo en la fosa de Panamá. Otros elementos morfológicos (Cresta de Cocos y Montes de Fisher) son responsables de las

Figura 4. Esquemas de las provincias-regiones fisiográficas y sísmicas de México

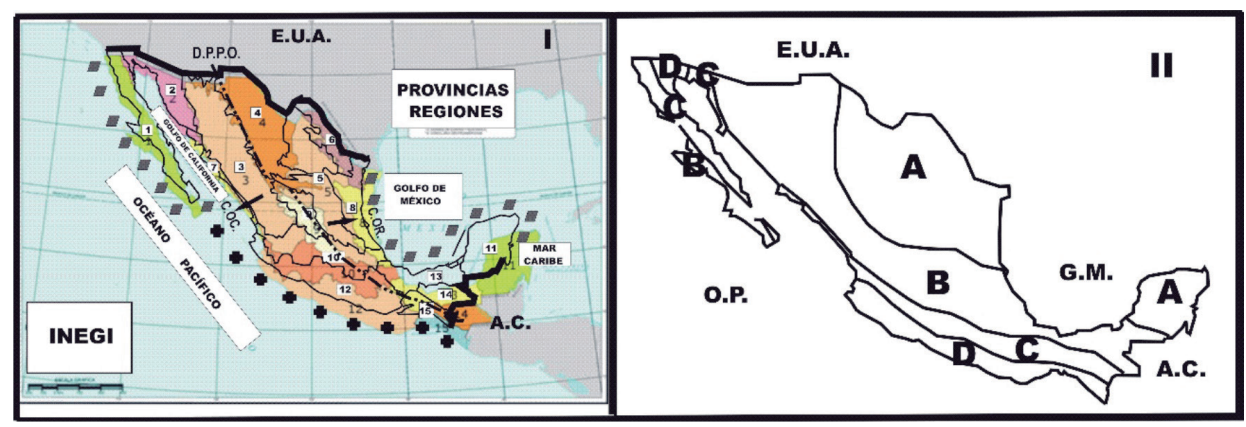

Aparecen: Lugares (A.C.= América Central, y E.U.A.= Estados Unidos de Norteamérica).

I: 1) Divisoria Principal de Primer Orden [D.P.P.O.] (líneas cortas negras y puntos); 2) Sentido del escurrimiento superficial (flechas negras) Cuenca [C.OCC. $=$ Occidental, C.OR. $=$ Oriental]; $\underline{\mathbf{3}}$ ) zonas de impacto de tsunamis de Fuentes (Lejanas (diamante color gris), Locales (cruz gruesa negra)); 4) Provincias fisiográficas ( $1=$ Península de la Baja California. $2=$ Llanura Sonorense, $3=$ Sierra Madre Occidental, $4=$ Sierras y Llanuras del Norte, $5=$ Sierra Madre Oriental, $6=$ Grandes Llanuras de Norteamérica, 7= Llanura Costera del Pacífico, 8=Llanura Costera del Golfo Norte, 9= Mesa del Centro, 10= Sierra Volcánica Transversal (Eje Neovolcánico), 11= Península de Yucatán, 12= Sierra Madre del Sur, 13= Llanura Costera del Golfo Sur, 14= Sierra de Chiapas y Guatemala, $15=$ Cordillera Centroamericana).

II Zonas sísmicas (nivel-categoría: $\mathrm{A}=\mathrm{Bajo}, \mathrm{B}=$ Medio, $\mathrm{C}=\mathrm{Alto}, \mathrm{D}=$ Muy alto). 
modificaciones de los planos de subducción, "asperezas". La profundidad de los terremotos en Costa Rica-Panamá es $<70 \mathrm{~km}$, el plano subducción $\sim 60^{\circ}$ y los de interior de placa $(\mathrm{h}>50 \mathrm{~km})$ y tienen con fallas normales.

\section{Relieve y Neotectónica}

La revisión bibliográfica sobre relieve y neotectónica (INEGI, 1981, 1990, 2005; Lugo, 1990; Ramírez Herrera, 2011) permite asegurar que: 1) hay 15 provincias fisiográficas (Figura 4-I) con 73 sub-provincias; 2) el sistema orográfico: 3 llanuras (Costera del Pacífico, Costera del Golfo y de la Península de Yucatán), una altiplanicie (mejicana), una depresión (Balsas) y un grupo de 6 cadenas-sierras montañosas (Baja California, Madre Occidental, Madre Oriental, Madre del Sur, Chiapas, Volcánica Transversal). La diferenciación longitudinal y transversal de los sistemas montañosos se relaciona con los esfuerzos desde las placas; 3) las líneas de costa occidental (I-IV) y oriental (V-IX) son heterogéneas y basculadas (Figura 1); 4) hay una cronología de terrazas marinas en las costas; 5) el sistema fluvial de superficie tiene una diferente organización y dimensión con 3 vertientes principales (Occidental, Oriental e Interior (situada entre las 2 anteriores, pero de menor dimensión)). Una divisoria principal de primer orden de aguas está en la figura 4-I.

Comparando las Provincias Geológicas con las Regiones Fisiográficas se constata una relación unívoca en la disposición de zonas y sectores. La influencia del sistema de placas se reconoce en: 1) el patrón de la sismicidad instrumental (Figura 5); 2) el mapa de Regiones Sísmicas (Figura 4-II); 3) la interrupción del sistema de zonas y regiones en el ENV. El sistema N-S está formado por 7 zonas (Llanura Costera del Pacífico, Sierra Madre Occidental, Sierras y Llanuras del Norte-Mesa del Centro, Grandes Llanuras de Norteamérica-Llanura Costera del Golfo Norte). La neotectónica vertical se evidencia en la costa del OP, donde hay diversos conjuntos de terrazas marinas emergidas (altitud 3-130 m), las zonas de: 1) PV (Punta Mita, Farallón, Tecuán, Manzanillo); 2) OX (Punta Maldonado, Brisa Zicatela, Santa Elena, Coyote); 3) PC. En la PC la tectónica determina al relieve, así: 1) el sistema de elevaciones posee escalones y asimetría transversal; 2) el desplazamiento lateral al NO relativo a la parte continental adyacente, a través de las fallas del Golfo, y tiene sismicidad. 
El contacto de placas (PT-1= Cerro Prieto-Laguna Salada (Punta Borrascosa)) donde la provincia de Salton Through conecta las fallas del Golfo de California y San Andrés (Figura 3). Hay cuencas "en-echelon y pullapart" y la de Salton se divide en: 1) Cerro Prieto (Mexicali, Baja California), 2) Brawley (Valle Imperial, California) y derivan desde el final del Mioceno Medio. En la parte emergida hay 3 zonas, todas de montaña: 1) $\mathrm{N}$ (dirección NNO), 2) Centro, igual dirección, y menos enérgico, 3) S, en contacto con las fallas del Golfo, que sugieren una ruptura continental.

La línea de costa de MX ha sido dividida en regiones (5 en el GMMar Caribe, 9 en el OP) por Lanza Espino et al. (2003). La macro-región del OP es más extensa y compleja que la Oriental, y distingue el cambio de estilo tectónico regional en las inmediaciones de Nayarit-PV, donde el ENV, pasa de transcurrente (PC) a subducción ( $\mathrm{S}$ de Nayarit). Ahí hay una zona de deformación neotectónica (Figuras 1 y 3). De modo que: 1) las provincias morfotectónicas resultan de la interacción de 3 placas (Norteamérica-Pacífico-Caribe) que han actuado simultáneamente durante el $\mathrm{Cr}$ Superior Tardío-Q; 2) los rasgos morfotectónicos observados en el continente y el margen Pacífico se asocian con fallas y fracturas (SO-NE). Ellas continúan activas y hay 14 intersecciones principales (conocidas como nudos) en la zona del OP y el interior. Aquí se asegura: 1) existe una zona de deformación activa, 2) las fallas están segmentadas, 3) hay más de 7 intersecciones en las inmediaciones de PV, y 3 en OX. Al comparar los territorios resulta que la primera posee mayor: 1) cantidad de fracturas, 2) número de zonas de articulación, 3) diversidad de direcciones, 4) actividad neotectónica. Hay una relación inversa en cuanto a las intersecciones $\mathrm{y}$ alineamientos entre las partes marina y continental $(\mathrm{PV}=7 / 4, \mathrm{OX}=3$ / 14). La AS en OX es mayor que en $P V$, y se explica por la dimensión de las placas y el diferente ángulo de convergencia. La figura 8 de Cotilla et al. (2017) muestra las deformaciones neotectónicas y los sistemas de fracturas y fallas en Nayarit-PV-Bahía de Banderas-Colima-Laguna de Chapala. Está comprobada la influencia de esfuerzos en lugares como el entorno del volcán Nevado-Toluca y NE de MX. Se prueba hay fa (E-O) y extensión (N-S), así como transcurrentes; y supone que entorno a PV hay una ligera tendencia de desplazamientos antihorario (al N) y horario (al S), por ser área de encuentro de las fallas del Golfo de California y la FM, con la intercalación en ángulo de la MPR. 
Mario Octavio Cotilla-Rodríguez - Diego Córdoba-Barba - Francisco Javier Núnez-Cornú

Caracterización Sismotectónica de México

\section{Datos de sismicidad}

La información de la sismicidad (IG, 2010; Medina, 1997; Nava, 1987; Núñez Cornú y Sánchez Mora, 1999; Núñez Cornú et al., 1998, 2002, 2004; Rutz López y Núñez Cornú, 2004; Suarez y Albini, 2009; SSN, 2000; Torres Vera, 2010) se resumen en las tablas 1, 3-10 y 15. En $1900-1990 \mathrm{MX}$ ha tenido $\sim 6.10^{3}$ muertos y pérdidas de valor $\sim 4.500 .10^{6}$ U.S.D. La figura 5 muestra la sismicidad del 2011 (4.168 epicentros) que se localiza mayoritariamente $(3.135(\sim 75 \%))$ en el OP, ajustada a la línea costera; pero su alineación, densidad y espaciamiento lateral es diferente por segmentos. La configuración tiene un mismo patrón sismotectónico que también está en los mapas de los años 2012-2018, y con valores de M 3-4 (78 \%), 4-5 (12\%), 5-7 (0,3\%). El mapa de sismicidad y cuerpos volcánicos de MX-AC de Benz et al. (2010) muestra para 1900-2010 la diferenciación espacio-temporal de la AS. Hay una banda estrecha y lineal de epicentros en color rojo en Golfo de California-AC, ajustada a la costa. Los eventos tienen $\sim 80 \mathrm{~km}$ de profundidad y los fuertes están agrupados en 2 segmentos principales: 1) PV-Guerrero (años: 1907, 1909, 1911, 1932, 1941, 1973, 1979, 1985, 1995, 2003), 2) OX (años: 1917, 1928, 1931, 1978, 1999). Predominan los terremotos con menores magnitudes y destacan la muy baja densidad en la PC y el Istmo de Tehuantepec; y la mayor AS está en AC. La disposición de terremotos más profundos (en color verde $(\mathrm{h}>70 \mathrm{~km})$ ) aparece a continuación de la banda anterior y hacia el interior. Se distingue la distorsión en cuanto a densidad de epicentros y geometría; y la banda es homogénea y densa (Veracruz-AC). Los terremotos del ENV son escasos, destacando el del 23.03.1908 M 7,5. El mapa tiene 2 isolíneas de profundidad de subducción (60-100 km), paralelas a la disposición de la línea de costa del OP; pero la isolínea de $100 \mathrm{~km}$ sólo está en Veracruz-AC.

En la FM se producen tsunamis que han afectado al litoral, y en ella se define la ZLP (Pacífico-Norteamérica-Caribe) donde se libera, a diferentes profundidades, la mayor cantidad de energía. Ella es una banda, relativamente homogénea y de 100-200 km de ancho, donde están la falla principal y otras de menores dimensiones (transversales y paralelas). También ocurren terremotos fuertes en el continente (interior de placa), pero no son frecuentes como los de tipo entreplacas con: 1) $\mathrm{M}>7,0 / \mathrm{h}>60$ $\mathrm{km} /$ mecanismo de falla normal (Chapala: 27.12.1568 M 7,5), hay de falla 
inversa (Ometepec: 20.03.2012 M 7,4); 2) $\mathrm{M}<7,0$ (Acambay: 19.11.1912 M 6,9; Jalapa: 03.01.1920 M 6,4); éstos ocasionan daños considerables; otros sismos con $\mathrm{M}<5,5$ se consideran sismicidad de fondo. Destaca que en 110 años (1907-2017) han ocurrido 8 terremotos con $\mathrm{M}>8,0$, y considerando la intensidad de XI grados (1754-2017) hay 9. Los valores de recurrencia son 14 y 29 años.

Tabla 3. Sismicidad de 1900-1995 (M> 7,5)

\begin{tabular}{|c|c|c|c|c|c|}
\hline M & Fecha & Coordenadas (N / O) & M & Fecha & Coordenadas $(\mathbf{N} / \mathbf{O})$ \\
\hline \multirow[t]{11}{*}{7,5} & 1902.04 .19 & $14,90 / 91,50$ & 7,7 & 1902.09 .23 & $18,00 / 106,50$ \\
\hline & 1908.03 .27 & $17,00 / 101,00$ & & 1911.06 .07 & $19,70 / 103,70$ \\
\hline & 1911.12.16 & $16,90 / 100,70$ & & 1917.12.29 & $15,00 / 97,00$ \\
\hline & 1928.03.22 & $16,23 / 95,45$ & & 1941.04 .15 & $18,85 / 102,94$ \\
\hline & .10 .09 & $16,30 / 97,30$ & & 1973.01.30 & $18,38 / 103,00$ \\
\hline & 1937.12 .23 & $17,10 / 98,07$ & 7,8 & 1928.06 .17 & $16,33 / 96,70$ \\
\hline & 1943.02.22 & $17,62 / 101,15$ & & 1932.06.18 & $19,50 / 103,50$ \\
\hline & 1957.07.28 & $17,11 / 99,10$ & & 1957.07.28 & $17,11 / 99,10$ \\
\hline & 1973.01 .30 & $18,39 / 103,21$ & & 1978.11.29 & $15,77 / 96,80$ \\
\hline & 1976.02.04 & $15,26 / 89,20$ & 7,9 & 1942.08 .06 & $14,80 / 91,30$ \\
\hline & 1985.09.21 & $17,62 / 101,82$ & & 1985.10 .09 & $18,79 / 104,47$ \\
\hline \multirow[t]{11}{*}{7,6} & 1903.01.04 & $15,00 / 93,00$ & 8,0 & 1907.04.15 & $16,70 / 99,20$ \\
\hline & 1911.06 .07 & $17,50 / 102,50$ & & 1931.01 .15 & $16,10 / 96,64$ \\
\hline & 1928.06 .17 & $16,33 / 96,70$ & & 1995.10.09 & $18,99 / 104,25$ \\
\hline & .10 .09 & $16,34 / 97,29$ & 8,1 & 1903.01.14 & $15,00 / 98,00$ \\
\hline & 1932.06.18 & $19,50 / 103,50$ & & 1908.03.26 & $16,70 / 99,20$ \\
\hline & 1941.04 .15 & $18,85 / 102,94$ & & 1985.09.19 & $18,14 / 102,71$ \\
\hline & 1965.08 .23 & $16,30 / 95,80$ & 8,2 & 1932.06 .03 & $19,57 / 104,82$ \\
\hline & 1973.01 .30 & $18,41 / 103,02$ & \multirow{4}{*}{\multicolumn{2}{|c|}{ Totales: }} & $7,5-7,9=33$ \\
\hline & 1978.11 .29 & $16,01 / 96,59$ & & & $8,0-8,2=7$ \\
\hline & 1979.03 .14 & $17,31 / 101,35$ & & & $1900-1960=28$ \\
\hline & 1985.09 .21 & $17,83 / 101,68$ & & & $1960-1995=13$ \\
\hline
\end{tabular}


Figura 5. Sismicidad registrada durante 2011 (SSN, Instituto de Geofísica, UNAM)

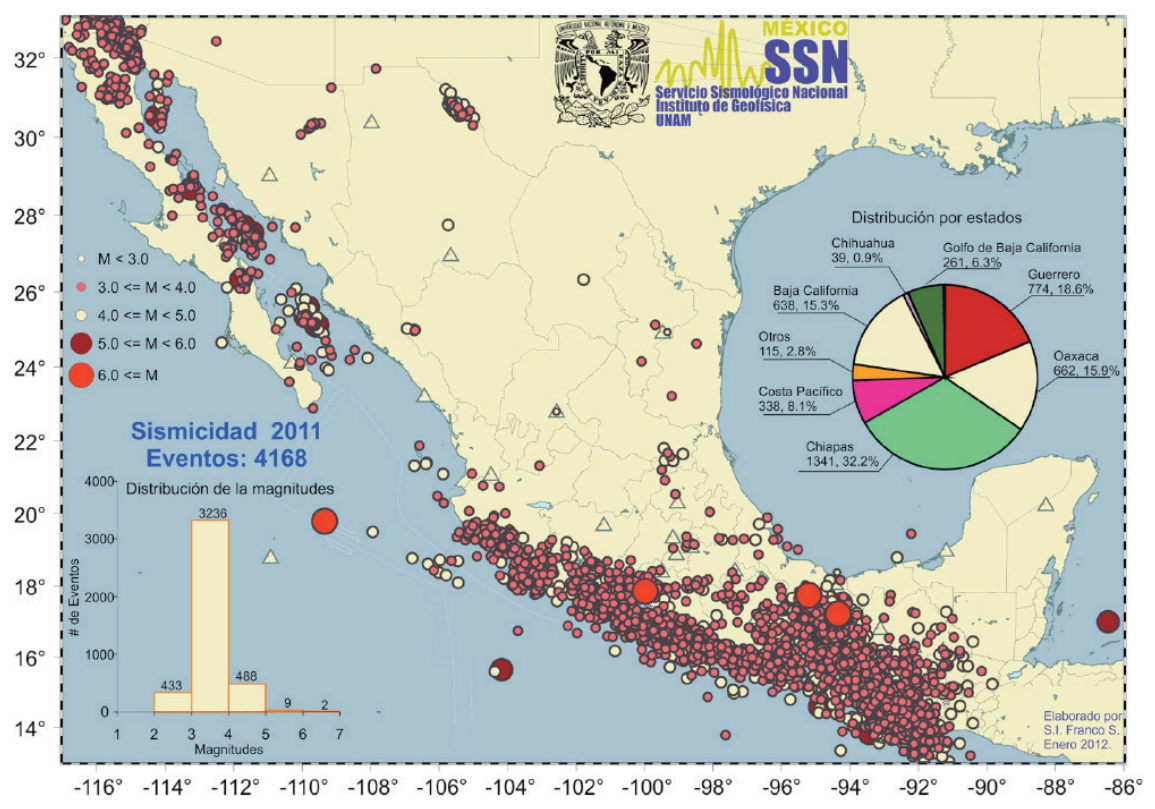

La tectónica activa se explica con estos terremotos: 1) 15.01.1931 Mw 8,0/ h $40 \mathrm{~km} /$ mecanismo focal de falla normal (E-O), en la ciudad de OX. El foco estuvo en la placa que subduce y bajo el continente; 2) los de 1932 en Jalisco $(03.06 \mathrm{Mw} 8,2$ y $18.06 \mathrm{Mw} 7,8)$ con una extensa zona de ruptura; 3) el del 11.12.1995 $\mathrm{Mw} 6,4$ con un plano de falla transcurrente derecho $\left(\mathrm{N} 87^{\circ} \mathrm{E}\right)$, en la zona de interacción de la MPR y la placa Cocos. Este es una réplica del terremoto del 09.10.1995 Mw 8,0. Todos están en la ZLP; 4) el del 07.09.2017 Mw 8,2 en el Golfo de Tehuantepec. Otros sismos que confirman la sismoactividad, diversa y distribuida, del territorio son: 1) los 4 terremotos de Veracruz (1-1) 03.01.1920 M 8,0, 1-2) 28.08.1973 M 7,7, 1-3) 07.04.2011 M 6,7, 1-4) 29.07.2014 M 6,4); 2) un sismo en Mexicali (Baja California) 04.04.2016 M 4,1; 3) 3 enjambres sísmicos en el Golfo de California (3-1) 1981 con 50 sismos, 3-2) 2007 con 91 terremotos, 3-3) 25-28.03.2016 con 47 sismos); 4) Guadalajara (Jalisco) 11.05.2016 4,8; 5) 2 terremotos en Quintana Roo (5-1) 10.06.2001 M 4,7, 5-2) 11.01.2015 M 4,2); 6) Chihuahua 27.08-09.2013 con 57 sismos 
de ellos 30 con $M>4,0$; 7) Saltillo (Coahuila) 31.05.2015 M 4,0; 8) 2 enjambres sísmicos en Nuevo León (8-1) 07.10.2013-18.03.2014 con más de 100 terremotos, 8-2) 2012 con 89 sismos). Con la sismicidad de 20172015, 2012-2011 por cantidad de ocurrencias en los Estados (Tabla 4A) se puede asegurar: 1) hay una mejora constante y sucesiva en la detección de sismos. Concretamente, hay 3 periodos: 1-1) 1990-1999 (8.315 (92 evento/año)); 1-2) 2000-2011 (82.857 (7.532 evento/año)); 1-3) 2012-2017 (70.650 (14.139 evento/año)); 2) que los mayores valores de AS están en 3 Estados del Pacífico (OX, Chiapas, Guerrero). Las cifras son: 2017 (85 \%), 2016 (75\%), 2015 (77\%), 2012 (78\%), 2011 (65\%); 3) que al incluir los Estados de Colima, Jalisco y Michoacán las cifras se incrementan 2017 (93\%), 2016 (89\%), 2015 (88\%); 4) que el terremoto más fuerte, del periodo, ocurrió en $\mathrm{OX}(8,2) ; 5)$ que predomina por cantidad el rango de $\mathrm{M}$ 6,0-6,9 (96 eventos) sobre el de 7,0-7,6 (16 eventos); 6) que la cantidad de terremotos en el intervalo de profundidad 8-60 km supera al de $>60-167$ $\mathrm{km}(18 / 4)$; 7) que sólo 2 eventos tienen $\mathrm{h}>100 \mathrm{~km} ; 8$ ) que las cantidades de terremotos con rango de M 7,5-7,9/ 8,0-8,2 de la tabla 1 (2000-2017) y la tabla 3 (1990-1995) son 1/ 1 y 40/ 7, respectivamente. En 1990-2017 los terremotos $(\mathrm{M}>6,0)$ representan $\sim 1 \%$ con promedio al año de $\sim 4$. La tabla 7A tiene la cantidad de terremotos por rangos de $\mathrm{M}$ del entorno del GM en 01.01.1999-31.01.2018.

Las figuras 6 y 7 de Pardo y Suárez (1995) presentan soluciones de los mecanismos focales y ejes $\mathrm{T}$, respectivamente; y dan una imagen de la tectónica del segmento PV-ENV-Chiapas, donde se aprecia la modificación de los planos de subducción. La figura 2 de Ego y Ansan (2002) recoge, para la parte central del ENV, 4 soluciones de mecanismos focales donde los ejes de esfuerzos principales indican direcciones NO-SE $\left(\sigma_{1}\right)$ y NE-SO $\left(\sigma_{2}\right)$. Esto se corresponde con los resultados sobre la extensión del Cuaternario y las soluciones de mecanismos (tipo compresivo) del tramo costero Manzanillo-Chiapas. El WSM de los años 2000-2016 permite comprobar, para el territorio de MX-AC-Caribe, lo comentado sobre los tensores y las direcciones de subducción, convergencia y contactos de placas. En la zona de interior de placa los mecanismos principales de la generación de terremotos son de 2 tipos: 1 ) debilidad-fragilidad cortical (por el proceso de subducción); 2 ) concentración de esfuerzos (por transmisión desde las placas). También la sismicidad en la placa Caribe, segmento de AC, tiene 
una importante densidad con profundidades $5-25 \mathrm{~km}$; algunos con Mmáx $>6,0$ aunque en Guatemala-Nicaragua hay valores mayores. Un centenar de terremotos fuertes está documentado en Honduras-Panamá, pero los más fuertes son de El Salvador (M 8,1) y los de menor nivel en Costa Rica (Tabla 2). Estas diferencias corresponden a variaciones del ángulo de los planos de subducción.

Tabla 4A. Sismicidad de México en 5 años (SSN-UNAM)

\begin{tabular}{|c|c|c|c|c|c|}
\hline Año (Total) & $\mathbf{2 0 1 7}$ & $\mathbf{2 0 1 6}$ & $\mathbf{2 0 1 5}$ & $\mathbf{2 0 1 2}$ & $\mathbf{2 0 1 1}$ \\
Estado & $\mathbf{( 2 6 . 4 1 3 )}$ & $\mathbf{( 1 5 . 4 6 0 )}$ & $\mathbf{( 1 0 . 9 4 6 )}$ & $\mathbf{( 5 . 0 8 1 )}$ & $\mathbf{( 4 . 1 6 8 )}$ \\
\hline Oaxaca (5) & $49,7 \%$ & $36,4 \%$ & $29,3 \%$ & $27,3 \%$ & $15,9 \%$ \\
Chiapas (11) & $22,9 \%$ & $18,9 \%$ & $29,3 \%$ & $26,4 \%$ & $32,2 \%$ \\
Guerrero (9) & $12,6 \%$ & $20,1 \%$ & $18,8 \%$ & $24,5 \%$ & $16,6 \%$ \\
Golfo de California (1-2) & $3,3 \%$ & $5,7 \%$ & $7,3 \%$ & $6,1 \%$ & $6,3 \%$ \\
\cline { 3 - 5 } Michoacán (8) & $3,4 \%$ & $5,6 \%$ & $5,8 \%$ & \multicolumn{1}{|}{} \\
Jalisco (6) & $2,5 \%$ & $4,3 \%$ & $2,0 \%$ & & \\
Colima (7) & $2,3 \%$ & $3,9 \%$ & $3,1 \%$ & & \\
Veracruz (16) & $1,7 \%$ & $2,8 \%$ & $2,2 \%$ & &
\end{tabular}

4B. Sismicidad de México (01.01.1999-31.01.2018) (SSN-UNAM)

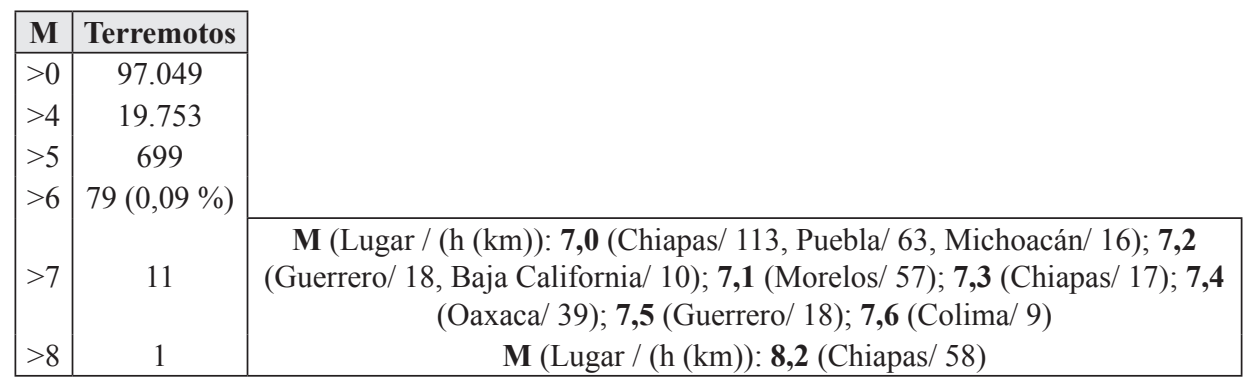

Tabla 5. Magnitudes (> 6,0) de México (1990-2017) (SSN-UNAM)

\begin{tabular}{|c|c|c|c|c|c|}
\hline Año & $\begin{array}{c}\text { Total } / \\
\text { Total }(M> \\
\mathbf{6 , 0})\end{array}$ & $\begin{array}{c}M(6,0-6,9) / M(7,0-7,9) / \\
M(>8,0)\end{array}$ & Año & $\begin{array}{c}\text { Total/ } \\
\text { Total }(M> \\
\mathbf{6 , 0 )}\end{array}$ & $\begin{array}{c}M(6,0-6,9) / M(7,0-7,9) / \\
M(>8,0)\end{array}$ \\
\hline 1990 & $796 / 2$ & $2 /-/-$ & 2004 & $1.346 / 3$ & $3 /-/-$ \\
\hline 91 & $728 / 1$ & $1 /-/-$ & 05 & $1.210 /-$ & $-1-1-$ \\
\hline 92 & $614 /-$ & $-1-1-$ & 06 & $1.355 /-$ & $-1-1-$ \\
\hline
\end{tabular}


Mario Octavio Cotilla-Rodríguez - Diego Córdoba-Barba - Francisco Javier Núnez-Cornú Seismotectonic characterization of Mexico

\begin{tabular}{|c|c|c|c|c|c|}
\hline Año & $\begin{array}{c}\text { Total/ } \\
\text { Total }(\mathbf{M}> \\
\mathbf{6 , 0})\end{array}$ & $\begin{array}{c}\mathbf{M}(\mathbf{6 , 0 - 6 , 9 ) / ~ M ~ ( 7 , 0 - 7 , 9 ) /} \\
\mathbf{M}(\mathbf{8 , 0 )}\end{array}$ & Año & $\begin{array}{c}\text { Total/ } \\
\text { Total }(\mathbf{M}> \\
\mathbf{6 , 0})\end{array}$ & $\begin{array}{c}\mathbf{M}(\mathbf{6 , 0 - 6 , 9 ) / ~ M ~ ( 7 , 0 - 7 , 9 ) / ~} \\
\mathbf{M}(\mathbf{8} \mathbf{8 , 0 )}\end{array}$ \\
\hline 93 & $916 / 6$ & $5 / 1 /-$ & 07 & $1.528 / 2$ & $2 /-/-$ \\
94 & $622 / 3$ & $3 /-/-$ & 08 & $1.955 / 2$ & $2 /-/-$ \\
95 & $678 / 9$ & $6 / 2 / 1$ & 09 & $2.302 / 1$ & $1 /-/-$ \\
96 & $789 / 3$ & $2 / 1 /-$ & 10 & $3.462 / 4$ & $3 / 1 /-$ \\
97 & $1.019 / 7$ & $6 / 1 /-$ & 11 & $4.292 / 5$ & $5 /-/-$ \\
98 & $1.024 / 5$ & $5 /-/-$ & 12 & $5.244 / 12$ & $10 / 2 /-$ \\
99 & $1.099 / 6$ & $4 / 2 /-$ & 13 & $5.360 / 4$ & $4 /-/-$ \\
2000 & $1.052 / 3$ & $2 / 1 /-$ & 14 & $7.607 / 8$ & $7 / 1 /-$ \\
01 & $1.344 / 6$ & $6 /-/-$ & 15 & $10.945 / 3$ & $3 /-/-$ \\
02 & $1.688 / 4$ & $3 / 1 /-$ & 16 & $15.281 / 6$ & $6 /-/-$ \\
03 & $1.323 / 4$ & $3 / 1 /-$ & 17 & $26.213 / 5$ & $2 / 2 / 1$ \\
\hline
\end{tabular}

El GM es un mar intra-continental $\left(\sim 1,5.10^{6} \mathrm{~km}^{2} /\right.$ profundidad máxima $\sim 4 \mathrm{~km}$ ) que está en una cuenca de tipo carbonatada con grandes espesores de sedimentos, donde hay un volumen importante de fluidos. En el SO han determinado deformaciones tectónicas activas con terremotos (Tablas 7A-B) y otros, de tipo interior de placa: 26.08.1959 18,26 N 94,43 E M 6,4 h (km) 21; 1967 19,23 N 95,74 O M 5,7 h (km) 26; 29.08.1973 M 8,3; 10.09.2006 26,32 N 86,84 E M 5,9 h (km) 30; 23.05.2007 21,98 N 96,14 E M 5,5 h (km) 44; 29.10.2009 19,14 N 95,58 E M 5,7 h (km) 17); y el segmento costero Tabasco-S de Veracruz, inmediato al Istmo de Tehuantepec, destaca por su AS ( $\mathrm{h}>100 \mathrm{~km})$, donde los eventos están relacionados con la subducción de la placa Cocos. Esto le diferencia del $\mathrm{N}$ del ENV, que tiene eventos someros. Este golfo es considerado, incorrectamente, un margen continental a-sísmico; pero se diferencia 3 sectores: 1) Costa Norte (Luisiana-Misisipi, con eventos débiles (24.07.1978 26,49 N 88,79 E M 5,0 h(km) 5); 2) Central, con eventos débiles (Mmáx 5,8); 3) SO, VeracruzCiudad del Carmen (con sismicidad superficial y terremotos de magnitudes bajas y medias (M 6,4)). Aquí la cantidad de terremotos registrados en los últimos 50 años es mayor que en los sectores $\mathrm{N}$ y Central. La AS del sector $\mathrm{N}$ está asociada con la sedimentación del río Misisipi. La concentración de terremotos en Ciudad del Carmen se debe a la extracción de fluidos. Lejos de la costa (Jaltiplan-Veracruz) han ocurrido algunos terremotos. 
Mario Octavio Cotilla-Rodríguez - Diego Córdoba-Barba - Francisco Javier Núnez-Cornú

Caracterización Sismotectónica de México

Tabla 6. Algunos terremotos en 4 Estados del oriente y centro de México

\begin{tabular}{|c|c|c|c|c|c|}
\hline Estado & Fecha & $\mathbf{M} / \mathbf{h}(\mathbf{k m}) /$ Fallecidos & Estado & Fecha & $\mathbf{M} / \mathbf{h}(\mathbf{k m})$ \\
\hline Veracruz & 1864.10 .03 & $7,3 /-$ & Tumaulipas & 2016.05 .16 & $3,6 / 5$ \\
& 1879.05 .09 & $7,0 /-$ & & .06 .17 & $3,7 / 10$ \\
& 1916.06 .02 & $7,0 / 150$ & & .01 .03 & $3,4 / 5$ \\
& 1920.01 .03 & $8,0 /-/>650$ & & .08 .15 & $3,6 / 10$ \\
& 1937.07 .25 & $7,7 / 85 / 34$ & & .15 & $4,0 / 10$ \\
& 1959.08 .26 & $6,4 / 21 / 20$ & & .09 .14 & $3,7 / 16$ \\
& 1973.08 .28 & $7,3 / 84 / 1.200$ & & .10 .21 & $3,5 / 10$ \\
Campeche & 2007.02 .09 & $4,8 / 10$ & & 2017.01 .17 & $3,7 / 10$ \\
& 2011.01 .23 & $4,4 / 10$ & Quintana Roo & .11 .13 & $3,9 / 10$ \\
& .02 .10 & $4,0 / 10$ & & 2002.06 .10 & $4,1 / 10$ \\
& 2015.05 .10 & $4,7 / 33$ & & 2015.01 .12 & $4,0 / 15$ \\
\cline { 2 - 6 } & 2016.03 .08 & $3,6 / 5$ & Totales: & $\mathbf{8 , 0 = 1}$ & $\mathbf{6 , 0 - 7 , 0 = 1}$ \\
& 2017.09 .25 & $4,0 / 15$ & & $\mathbf{7 , 0 - 7 , 9 = 5}$ & $\mathbf{3 , 0 - 6 , 0 = 1 7}$ \\
\hline
\end{tabular}

La figura 1 de Franco et al. (2013) muestra agrupamiento de terremotos en la intersección ENV-GM, donde predomina la AS de baja energía y poca profundidad $(<70 \mathrm{~km})$ y esto coincide en el volcán San Andrés Tuxla. Otros epicentros más escasos y dispersos están en Xalapa-Tampico y Veracruz-Campeche. En este último hay una importante cantidad (h>70 $\mathrm{km})$. Ramos Zúñiga et al. (2012) presentan en su figura 1 un grupo de 7 mecanismos focales incluidos en un fondo de sismicidad (1847-2012) para las partes $\mathrm{N}$ y oriental de MX y del S de U.S.A. (Texas). La sismicidad, de mayor energía, tiene desplazamiento temporal del O al E: 1) en la zona de Texas, con igual mecanismo (tipo normal) hay 3 terremotos: 16.08.1931 (6,4/ $10 \mathrm{~km} /$ Valentine), 14.04.1995 (5,7/ $17 \mathrm{~km} /$ Alpine), $20.10 .2011(4,8 /$ $3 \mathrm{~km} /$ San Antonio); 2) en la zona de MX están 4 eventos: 01.11 .1928 (6,5/ 10 km/ Parral, Durango), 17.04.2006 (3,3/ 20 km Santiago, Nuevo León), 14.06.2009 (5,0/20 km/ General Terán, Nuevo León), 14.06.2009 (5,1/ 10 $\mathrm{km} /$ China, Nuevo León). Los 2 primeros tienen mecanismo normal y los de 2009, muy cercanos entre sí, son transcurrentes; 3) el tiempo de ocurrencia es similar (Texas $=80$ años, $M X=81$ años $)$; 4) la distancia entre los primeros eventos, en las bandas respectivas, y los últimos es aproximadamente la misma. Esto permite asumir que los terremotos son de una misma familia ST. La profundidad de los eventos (2006-2007) determinados por una red local temporal en Santiaguillo (Estado de Durango) es $<2,5$ y $<10$ $\mathrm{km}$, respectivamente. Esto se corresponde con la tectónica contemporánea y la sismicidad de interior de placa. En la figura 2 de Franco et al. (2013) 
hay 10 soluciones de mecanismos focales, que ilustran la dinámica del GM. Ellas corresponden a fallas inversas (dirección NO-SE/ buzamiento $45^{\circ}$ ) y se asocian con los procesos de sedimentación. Otras soluciones de mecanismos (23.05.2007 del margen occidental) están relacionados con la falla Oriental Mejicana y un régimen transformante. Singh et al. (2015) presentan 7 mecanismos (26.08.1959 M 6,4 h(km) 21, 11.03.1967 M 5,7 h(km) 26, 24.07.1978 M 5,0 h(km) 15, 06.09.1997 M 4,5 h(km) 30, 10.09.2006 M 4,9 h(km) 30, 23.03.2007 M 5,6 h(km) 24, 29.10.2009 M $5,4 \mathrm{~h}(\mathrm{~km}) \mathrm{17})$ y llegan a conclusiones equivalentes.

Tabla 7A. Terremotos del Golfo de México 01.01.1999-31.01.2018 (SSN-UNAM)

\begin{tabular}{|c|c|c|c|c|c|}
\hline Fecha & $\begin{array}{c}\mathrm{M} / \mathbf{h}(\mathbf{k m}) / \text { Coorde- } \\
\text { nadas }(\mathrm{N} / \mathrm{O})\end{array}$ & Estado & Fecha & $\begin{array}{c}\text { M/h(km)/ Coorde- } \\
\text { nadas (N/O) }\end{array}$ & Estado \\
\hline 2006.09 .10 & $5,7 / 10 / 26,25 / 87,26$ & \multirow[t]{10}{*}{ Q.Roo } & 1993.08 .23 & $4,8 / 10 / 22,38 / 99,23$ & \multirow[t]{10}{*}{ Tamaulipas } \\
\hline 2003.04 .10 & $5,4 / 10 / 15,87 / 87,95$ & & 1992.11 .30 & $4,8 / 30 / 23,26 / 98,03$ & \\
\hline 2011.12 .10 & $4,8 / 34 / 16,87 / 86,18$ & & 2008.09 .23 & $4,3 / 12 / 22,45 / 97,57$ & \\
\hline 2013.08 .24 & $4,7 / 10 / 15,57 / 86,04$ & & 1994.04.09 & $4,3 / 12 / 23,12 / 99,65$ & \\
\hline 2012.05 .03 & $4,7 / 20 / 17,27 / 85,55$ & & 2015.08 .04 & $4,1 / 5 / 24,31 / 99,04$ & \\
\hline 2002.06 .10 & $4,6 / 12 / 19,04 / 88,08$ & & 2012.06 .24 & $4,1 / 16 / 22,64 / 97,99$ & \\
\hline 2008.06 .21 & $4,5 / 20 / 17,96 / 87,87$ & & 2008.10 .30 & $4,1 / 25 / 22,54 / 97,83$ & \\
\hline 2012.05.11 & $4,4 / 10 / 15,83 / 86,87$ & & 2016.08 .14 & $4,0 / 10 / 24,24 / 98,54$ & \\
\hline 2014.01.13 & $4,3 / 82 / 16,23 / 88,67$ & & 2015.08 .07 & $4,0 / 3 / 24,27 / 99,29$ & \\
\hline 2015.01.11 & $4,2 / 20 / 20,32 / 87,51$ & & 2011.02 .12 & $4,0 / 35 / 23,18 / 99,15$ & \\
\hline 2008.02.09 & $4,8 / 20 / 19,60 / 92,25$ & \multirow[t]{7}{*}{ Tabasco } & 1999.06.15 & $7,0 / 63 / 18,13 / 97,54$ & \multirow[t]{5}{*}{ Veracruz } \\
\hline 2016.03.08 & $4,7 / 33 / 19,54 / 92.17$ & & 2011.04 .07 & $6,7 / 171 / 17,22 / 94,32$ & \\
\hline 2015.05 .10 & $4,5 / 13 / 19,60 / 92,25$ & & 2014.07.29 & $6,4 / 117 / 17,70 / 95,67$ & \\
\hline 2005.04 .01 & $4,5 / 18 / 18,74 / 92,04$ & & 2011.02 .25 & $6,0 / 135 / 17,69 / 95,21$ & \\
\hline 1998.06 .03 & $4,4 / 34 / 19,04 / 90,84$ & & 2004.06 .29 & $4,2 / 10 / 27,91 / 90,18$ & \\
\hline 2013.10 .20 & $4,3 / 5 / 19,51 / 92,29$ & & .06 .22 & $4,1 / 114 / 19,42 / 88,82$ & \multirow[t]{2}{*}{ Yucatán } \\
\hline 2011.01 .22 & $4,3 / 10 / 19,50 / 92,12$ & & 2015.04 .07 & $2,9 / 10 / 21,22 / 89,40$ & \\
\hline 2000.01 .14 & $4,3 / 10 / 19,46 / 92,01$ & Totales: & $6,0-7,0=1$ & $5,0-6,0=5(16 \%)$ & $\begin{array}{c}2,9-5,0= \\
31\end{array}$ \\
\hline 2001.07.20 & $4,2 / 156 / 19,04 / 92,05$ & & & & \\
\hline 1998.06 .03 & $4,0 / 34 / 19,04 / 90,84$ & & & & \\
\hline
\end{tabular}


Mario Octavio Cotilla-Rodríguez - Diego Córdoba-Barba - Francisco Javier Núnez-Cornú Caracterización Sismotectónica de México

7B. Cantidad de terremotos en el entorno del Golfo de México (1990-2017)

\begin{tabular}{|c|c|c|}
\hline Estado & M & Terremotos \\
\hline Veracruz (16) & $>0 />4,0$ & $1.941(86 \%) / 754$ \\
Tabasco (15) & $>0$ & 142 \\
Yucatán (13) & $>0$ & 82 \\
Tamaulipas (18) & $>0$ & 78 \\
Quintana Roo (12) & $>0$ & 11 \\
\hline
\end{tabular}

La tabla 8 tiene datos de la sismicidad (1812-1984) en PC-Golfo de California, y en las tablas 9A-B hay 44 determinados por el SSN. Se ve que: 1) la Mmáx es menor a 7,2; 2) las profundidades son inferiores a 15 $\mathrm{km}$. La figura 1B de Márquez Azua et al. (2004) contiene epicentros de la: A) margen occidental mejicana desde Baja California a Chiapas. Destacan la: 1) alineación costera de los epicentros; 2) muy diferente densidad de epicentros (mucho menor en Baja California); B) zona del GM. En esta la sismicidad está muy dispersa y su densidad es mucho menor que en el margen Pacífico. Se estima que en el GM y la PC ocurren 132 y 325 terremotos/ año, respectivamente. La región produce 18 eventos con M 2,9-7,0/ año (18.06.1973 M 7,0; 04.01.2006 M 6,7 h 10 km; 19.01.2018 M 6,3 h $16 \mathrm{~km}$ 10:17:45 26,66 N 111,10 O 11 réplicas) en Loreto. Esto confirma la mayor AS de la PC y el Golfo de California, y con la sismicidad histórica y los datos de la tabla 14 se asegura que Baja California Norte es más activa que la Sur.

Tabla 8. Terremotos en la Península de California (1812-1984)

\begin{tabular}{|c|c|c|c|}
\hline Zona & Fecha & M/h(km) & Observaciones \\
\hline N de Tecate & 1812.12 .08 & $7,5 /-$ & Evento de San Juan Capistrano-40 muertos \\
N Sonora & 1887.05 .03 & $7,4 /-$ & Evento de Bavispe-51 fallecidos \\
\cline { 4 - 4 } B. California & 1901.12 .08 & $7,0 /-$ & \\
California & 1902.12 .12 & $7,1 /-$ & \\
G. California & 1907.10 .16 & $7,1 / 10$ & \\
G. California & 1915.11 .21 & $7,0 / 10$ & \\
B. California & 1932.12 .31 & $7,1 /-$ & \\
- & 1934.12 .12 & $7,1 /-$ & \\
B. California & 1940.05 .19 & $7,1 /-$ & \\
& & &
\end{tabular}




\begin{tabular}{|c|c|c|c|}
\hline Zona & Fecha & M/h(km) & Observaciones \\
\hline G. California & 1954.04 .20 & $7,0 /-$ & \\
- & 1956.02 .09 & $6,7 /-$ & \\
- & 1979.11 .21 & $7,0 /-$ & \\
- & 1980.06 .09 & $6,1 /-$ & Evento del Valle Imperial \\
- & 1987.11 .24 & $6,5 /-$ & \\
& &
\end{tabular}

Las informaciones sobre zonas sísmicas y peligrosidad de Esteva (1970), INEGI (2005A), Zúñiga et al. (1997). Las 3 gráficas de estos últimos: No 3.1 (Sismicidad en base al catálogo 1900-1994), No 4.1a (Temblores de subducción), $\mathrm{N}^{\mathrm{o}} 4.1 \mathrm{~b}$ (Temblores de profundidad intermedia) se corresponden con las determinaciones del SSN. Esto confirma que: 1) MX es un País con peligro sísmico; 2) los periodos de recurrencia, de terremotos fuertes, aunque diferentes por regiones; 3) algunas estructuras sismogenéticas tienen relación de continuidad en los países vecinos. Las figuras 2.2 y 2.3 de RESIS II (2008) tienen las soluciones de mecanismos focales (1976-2007) para AC $\left(\mathrm{Mw}>6,011^{\circ}-19^{\circ} \mathrm{N} 82^{\circ}-95^{\circ} \mathrm{O}\right.$ y $\mathrm{Mw}>5,506^{\circ}-13^{\circ} \mathrm{N}$ $\left.76^{\circ}-88^{\circ} \mathrm{O}\right)$. En la primera son de tipo normal en la Depresión de Honduras (parte N) y transcurrentes de izquierda (Swan y Motagua). Para la segunda figura destaca la alineación N-S de los mecanismos tipo transcurrente asociados con la Zona de Fracturas de Panamá. Del SSN se conoce del terremoto de 20.03.2012 que: 1) el sismo principal tuvo: M 7,4 h $15 \mathrm{~km} \mathrm{12:02}$ horas 16,42 N 98,36 O (en las cercanías de Ometepec-Guerrero-Pinotepa Nacional, OX)/ sentido en gran parte de la zona centro de MX; 2) en el intervalo temporal 23-26.03 hubo 193 réplicas con M 3,3-5,3 y h (km) $1-24 ; 3)$ algunos de los terremotos más importantes en el Estado de OX están relacionados con: 3-1) la placa de Norteamérica (15.01.1931 M 8,0; 02.08.1968 M 7,3; 30.09.1999 M 7,6); 3-2) el proceso de subducción de la placa Cocos (23.08.1965 M 7,5; 29.11.1978 M 7,6); 4) las localidades que históricamente han sido más afectadas por sismos son Puerto Escondido, Pochutla, Puerto Ángel, Huatulco, Loxitla, Cacahua, Jamiltepec, Pinotepa Nacional, Ometepec y Miahuatlan. El terremoto del 2012 coincide con el del 16.02.2017 M 7,2 (Figura 1). En 2015-2017 el SSN ha indicado 13 terremotos importantes (3 (2015), 6 (2016), 4 (2017)) (Tabla 5), de ellos uno (13.09.2015 M 6,7) está en Baja California Sur, el resto al S de Jalisco, y el de mayor $\mathrm{M}$ en $\mathrm{OX}$. 
Mario Octavio Cotilla-Rodríguez - Diego Córdoba-Barba - Francisco Javier Núnez-Cornú

Caracterización Sismotectónica de México

Tabla 9A. Terremotos de Baja California y Baja California Sur (01.01.1999-31.01.2018) (SSN-UNAM)

\begin{tabular}{|c|c|c|c|}
\hline Estado & Baja California Norte (1)= 4 Fallecidos & Estado & \multicolumn{1}{c}{ Baja California Sur (2) } \\
\hline Fecha & $\mathbf{M} / \mathbf{h}(\mathbf{k m}) /$ Coordenadas (N/ O) & Fecha & M / h(km) / Coordenadas (N/ O) \\
\hline 2010.04 .04 & $7,2 / 10 / 32,47 / 115,37$ & 2012.04 .12 & $6,8 / 5 / 28,83 / 113,16$ \\
2012.12 .14 & $5,4 / 15 / 31,19 / 119,42$ & 2015.09 .13 & $6,7 / 8 / 25,04 / 109,53$ \\
2014.03 .21 & $5,6 / 17 / 30,39 / 114,08$ & 2007.09 .01 & $6,5 / 2 / 24,91 / 109,97$ \\
2013.08 .26 & $5,6 / 17 / 33,09 / 115,47$ & 2010.10 .21 & $6,5 / 67 / 24,34 / 106,69$ \\
2012.08 .22 & $5,5 / 16 / 23,27 / 115,50$ & 2018.01 .19 & $6,3 / 16 / 26,22 / 111,10$ \\
2002.02 .22 & $5,5 / 5 / 32,20 / 115,44$ & 2013.10 .19 & $6,3 / 14 / 26,09 / 110,46$ \\
2016.03 .27 & $5,4 / 15 / 30,00 / 114,50$ & 2007.09 .01 & $6,3 / 2 / 24,91 / 109,97$ \\
2010.04 .08 & $5,4 / 10 / 32,33 / 115,10$ & 2012.09 .25 & $6,3 / 15 / 24,61 / 110,57$ \\
2014.03 .21 & $5,3 / 20 / 30,25 / 114,13$ & 2002.10 .03 & $6,2 / 35 / 23,22 / 108,73$ \\
2008.02 .11 & $5,3 / 16 / 32,14 / 115,00$ & 2014.10 .07 & $6,1 / 10 / 23,70 / 108,76$ \\
2014.09 .15 & $5,2 / 5 / 30,214 / 114,32$ & 2012.04 .12 & $6,0 / 15 / 28,80 / 113,41$ \\
2011.12 .23 & $5,2 / 20 / 30,52 / 114,27$ & 2011.11 .01 & $6,0 / 16 / 19,73 / 109,30$ \\
2014.03 .21 & $5,1 / 5 / 30,42 / 114,02$ & .01 & $6,0 / 15 / 19,74 / 109,31$ \\
.04 & $5,1 / 15 / 31,19 / 119,42$ & 2012.10 .08 & $5,8 / 14 / 20,12 / 109,94$ \\
2011.02 .18 & $5,1 / 20 / 32,14 / 115,01$ & 2013.01 .13 & $5,7 / 10 / 25,92 / 110,34$ \\
2010.09 .50 & $5,1 / 10 / 29,90 / 114,18$ & 2012.10 .05 & $5,5 / 10 / 23,58 / 108,51$ \\
2004.08 .30 & $5,1 / 10 / 29,52 / 116,55$ & 2011.04 .26 & $5,4 / 18 / 27,85 / 111,39$ \\
2011.02 .20 & $5,0 / 15 / 32,23 / 115,14$ & 2012.04 .15 & $5,4 / 15 / 28,94 / 113,22$ \\
2010.09 .14 & $5,0 / 10 / 32,17 / 115,08$ & .09 .13 & $5,3 / 28 / 24,90 / 109,42$ \\
2005.04 .26 & $5,0 / 10 / 30,25 / 114,13$ & .04 .12 & $5,2 / 16 / 24,12 / 108,97$ \\
2003.02 .07 & $5,0 / 8 / 31,63 / 115,14$ & 2015.09 .13 & $6,7 / 100 / 25,04 / 109,53$ \\
1999.06 .03 & $5,0 / 5 / 32,37 / 115,22$ & .26 & $5,0 / 20 / 24,13 / 108,93$ \\
\hline
\end{tabular}

9B. Cantidad de terremotos en el entorno de P. de California (1900-2017)

\begin{tabular}{|c|c|c|}
\hline Estado & M & Terremotos \\
\hline Baja California (1) & $>0 />4,0 />5,0$ & $3.713(67 \%) / 514 / 26$ \\
\hline Baja California Sur $(2)$ & $>0 />4,0 />5,0 />6,0$ & $1.818(33 \%) / 517 / 63 / 12$ \\
\hline
\end{tabular}

Tabla 10. Selección de terremotos del periodo 2015-2017

\begin{tabular}{|c|c|c|c|}
\hline Fecha / Tiempo & $\begin{array}{c}\text { M / h(km) / Coordenadas } \\
\text { (N / O) }\end{array}$ & Fecha / Tiempo & $\begin{array}{c}\mathbf{M} / \mathbf{h}(\mathbf{k m}) / \text { Coordenadas } \\
\text { (N / O) }\end{array}$ \\
\hline 2015.02.22/08:23:16 & $6,2 / 16 / 18,64 / 106,96$ & $2016.05 .08 / 02: 33: 59$ & $6,0 / 35 / 16,25 / 97,98$ \\
$.09 .13 / 03: 14: 09$ & $6,7 / 8 / 25,04 / 109,53$ & $.06 .07 / 05: 51: 36$ & $6,1 / 16 / 18,23 / 105,38$ \\
$.12 .17 / 13: 49: 54$ & $6,6 / 91 / 15,76 / 93,70$ & $2017.06 .14 / 02: 29: 05$ & $7,0 / 107 / 17,73 / 92,32$ \\
$2016.01 .21 / 12: 06: 58$ & $6,5 / 10 / 18,79 / 107,15$ & $.07 .09 / 23: 49: 17$ & $8,2 / 46 / 14,76 / 94,10$
\end{tabular}




\begin{tabular}{|l|l|l|l|}
$.04 .15 / 09: 11: 25$ & $6,1 / 12 / 13,56 / 92,28$ & $.19 / 13: 14: 41$ & $7,1 / 39 / 18,34 / 98,68$ \\
$.25 / 02: 07: 09$ & $6,0 / 16 / 14,35 / 93,26$ & $.23 / 07: 53: 04$ & $6,1 / 11 / 16,50 / 95,15$ \\
\cline { 4 - 5 } $.27 / 07: 51: 19$ & $6,0 / 20 / 14,35 / 93,26$ & \multicolumn{2}{|l}{} \\
\cline { 3 - 4 }
\end{tabular}

La tabla 11A tiene datos instrumentales de terremotos de América con más de 1.000 fallecidos y 2 de México D.F. y Veracruz. Datos de los eventos más fuertes que afectaron América y Japón están en la tabla 11B. La energía liberada es elevada y tales valores no están en MX. Los datos utilizados sobre tsunamis y sus peligros (Soloviev, 1970; Lockridge y Smith, 1984; Sánchez y Farreras, 1993; Kuroiwa, 1995; Farreras, 1997; Arce et al., 1998; Gusiakov, 2002; NOAA, 2018; Brink et al., 2009; SEMAR, 2012; Geophysics Data Center, 2017) permiten asegurar que: hay 2 tipos de fuentes tsunamigénicas en el territorio: 1) cercanas (o locales), 2) lejanas (Figura 4-I). La información de 1754-2018 indica el impacto de $\sim 50$, los tsunamis en la costa del Pacífico de MX. De ellos hay 230 de fuentes locales, con olas 0,1-10 m (Tabla 12A). Los efectos están en $\mathrm{N}$ de PV-AC, y hay diferencias entre la altura máxima de las olas por registro instrumental ( $3 \mathrm{~m}$ ) y los datos históricos (mucho mayores). Hay una selección de tsunamis de 10 fuentes lejanas que han afectado las costas mejicanas (Tabla 12B). El rango de altura de las olas es 0,2-2,5 $\mathrm{m}$ y la frecuencia es mayor que la de los tsunamis locales.

Tabla 11A. Selección de terremotos de América con más de mil fallecidos

\begin{tabular}{|c|c|c|c|c|c|c|c|}
\hline Fecha & M & Fallecidos & País & Fecha & M & Fallecidos & País \\
\hline 2010.01 .12 & 7,0 & 300.000 & Haití & 1985.09 .19 & 8,1 & 3.150 & México \\
1970.05 .31 & 7,9 & 50.000 & Perú & 1906.04 .17 & $7,9-8,6$ & $478-3.000$ & U.S.A. \\
1939.01 .24 & 7,8 & 30.000 & Chile & 1986.10 .10 & 7,5 & 2.000 & El Salvador \\
1976.02 .04 & 7,9 & 22.870 & Guatemala & 1999.01 .25 & 6,2 & 1.230 & Colombia \\
1972.12 .23 & 6,2 & 20.000 & Nicaragua & 1973.08 .28 & 7,3 & 1.200 & México \\
\hline
\end{tabular}

11B. Selección de terremotos y tsunamis devastadores

\begin{tabular}{|c|c|c|}
\hline Fecha & M (terremoto) / Hmáx olas (m) & País \\
\hline 1964.03 .27 & $9,2 / 70$ & U.S.A. (Alaska) \\
2010.02 .27 & $8,8-9,0 / 10$ & Chile \\
2011.03 .11 & $9,1 / 40,5$ & Japón \\
\hline
\end{tabular}


Mario Octavio Cotilla-Rodríguez - Diego Córdoba-Barba - Francisco Javier Núnez-Cornú Caracterización Sismotectónica de México

Tabla 12A. Mayores tsunamis locales de México (1754-2017)

\begin{tabular}{|c|c|c|c|c|}
\hline Fecha & M / I (Mercalli) & Altura (m) & Región & Fallecidos \\
\hline 1754.09 .01 & $-/$ XI & 4 & Guerrero & - \\
1787.03 .28 & $8,3 /$ IX & 4 & Guerrero-Oaxaca & 11 \\
1928.06 .17 & $7,8 /-$ & - & Oaxaca & - \\
1932.06 .03 & $8,2 /-$ & 10 & Jalisco & 4 \\
.22 & $7,0 /-$ & 10 & Jalisco & 75 \\
1962.05 .11 & $7,1 /-$ & 5 & Guerrero & - \\
1985.09 .19 & $8.0 /-$ & 2 & Michoacán & - \\
1995.10 .19 & $8,0 /-$ & 5 & Michoacán & 1 \\
\hline
\end{tabular}

12B. Tsunamis de fuentes lejanas

\begin{tabular}{|c|c|c|c|c|}
\hline Fuente & Fecha & M & Altura (m) & Región afectada \\
\hline Alaska & 1964.03 .20 & 9,2 & 2,4 & Baja California \\
Chile & 1960.05 .22 & $8,8-9,0$ & 2,5 & Baja California \\
Colombia & 1979.12 .12 & 7,9 & 0,3 & Guerrero \\
Hawai & 1975.11 .29 & 7,2 & 0,5 & Baja California \\
& 1967.03 .09 & 8,3 & 1,0 & Baja California \\
Islas Aleutinas & 1965.02 .04 & 8,2 & 0,5 & Oaxaca \\
Islas Kuriles & 1963.10 .13 & 8,1 & 0,5 & Guerrero \\
Japón & 1968.05 .16 & 8,0 & 0,3 & Baja California \\
Kamchatka & 1952.11 .04 & 8,3 & 1,2 & Oaxaca \\
Kermadec & 1976.01 .14 & 7,3 & 0,2 & Guerrero \\
& .11 .20 & 6,8 & 0,1 & Guerrero \\
Perú & 1966.10 .17 & 7,5 & 0,2 & Oaxaca \\
\hline
\end{tabular}

Las costas tienen 3 zonas con relación a los tsunamis: 1) la del OP con 2 zonas (una como receptora (Baja California) y otra generadora de tsunamis (Nayarit-Chiapas)); 2) la del GM es receptora (indicada por primera vez) (Figura 4-I). Hay 4 segmentos costeros que tienen importantes áreas de peligro por desprendimientos rocosos, gravedad, e impacto de tsunamis. Ellos están asociados a las zonas físico-geográficas 1 (Península Baja California), 10 (Sierra Volcánica Transversal), 12 (Sierra Madre del Sur) (Figura 4-I) y a las zonas sísmicas B, C, D (Figura 4-II). Se asegura que en: 1) AC se han producido 49 tsunamis (periodo 1539-2000); de ellos 37 son del OP y el resto del Caribe. La cifra es equivalente a la de MX; 2) En MX no han ocurrido eventos como los de la tabla 11B. 


\section{Discusión y conclusiones}

Los datos de Azuz Adeath y Rivera Arriaga (2009) muestran que en el periodo de 2000-2005: 1) son 17 Estados los que tienen relación directa con la mar; 2) que la población pasó de 10.848 .221 a 11.951 .969 en la zona del OP-Mar de Cortés; 3) de los 265 municipios costeros, 92 tienen apertura al mar, 31 influencia marina, 32 influencia marina alta-media y 89 influencia marina alta. El SPNM (2017) dice que son 19 puertos deportivos, 15 de ellos están en el OP y 4 en el GM. Hay 4 instalaciones que reciben cruceros en: Cabo San Lucas, Ensenada, Mazatlán y PV; además, son 117 Puertos y Terminales Portuarias con importantes movimientos de personas, y puestos de trabajo. MX tiene 3 tipos de peligros (terremotos, volcanes y tsunamis) en ocasiones interrelacionados. Ellos han afectado a la población y sus infraestructuras. La práctica ST demuestra que en regiones de alta AS no siempre es factible relacionar focos con las fallas conocidas. En la página 17 del Informe del SGM (2007) se encuentra una figura con 15 Corredores Sismotectónicos para los Estados de OX-Chiapas-Tabasco. Ellas son bandas irregulares, en general alargadas y con direcciones muy diferentes, que no tienen relación aparente con la estructura neotectónica. El uso de estos elementos (alineamientos y zonas sismotectónicas) se encuentra en Armenia, California, Cuba, España, La Española, Mar de Barents, Rumanía y Rusia. Se considera que las zonas de debilidad tectónica pre-existentes, en el interior de placa, determinan la ocurrencia de los terremotos, como se demuestra en U.S.A., MX y Cuba (Cotilla Rodríguez, 2017). La hipótesis relaciona la heterogeneidad de la corteza con la distribución espacial de los terremotos en un marco tensorial por transmisión de los esfuerzos desde fuentes alejadas. Otra vía está en el ejemplo de OX, para un intervalo temporal de 8 años, por 3 grupos distintos (Núñez Cornú y Ponce, 1989). Todos se fundamentan en la sismicidad, pero dividen diferentemente el territorio en 3, 5 y 8 zonas sísmicas. Éstas cubren la superficie emergida y colindan entre sí, y tienen configuraciones diferentes, aunque mantienen un paralelismo aproximado con la línea de costa.

Hay 5 tipos de terremotos en MX que están en las placas que interaccionan y se corresponden con los 2 tipos de sismicidad. En un perfil O-E (OP-GM) los terremotos son de tipo: 1) ante-fosa (la FM), asociados a la zona inmediata a la deformación o curvatura de la placa Cocos bajo la placa de Norteamérica. Los eventos corticales son del interior de la placa 
oceánica, y con magnitudes bajas, y no producen tsunamis; 2) entreplacas, que corresponden a la interacción de las placas Cocos-Norteamérica, y la MPR. Ellos ocurren en el contacto-acoplamiento $(\mathrm{h}<20-30 \mathrm{~km})$ y tienen magnitudes de 6,0-8,2 y pueden producir tsunamis; 3) falla transcurrente (desplazamiento lateral), en el contacto: 3-1) placa Cocos-MPR; 3-2) placas Pacífico-Norteamérica; 3-3) placas Norteamérica-Cocos-Caribe. Éstos no generan tsunamis $\mathrm{M} 5-7,9)$; 4) de interior de la placa Cocos, cuando ésta subduce bajo la de Norteamérica, con hipocentros de 50-200 km M 8,0 . Los efectos son muy importantes en la zona continental mejicana; 5) de interior de la placa de Norteamérica, donde las profundidades son $<20$ $\mathrm{km}$. Predominan las magnitudes bajas, y los pocos terremotos fuertes que causan graves pérdidas. Los elementos de ST se definen por: 1) las características del entorno tectónico; 2) los elementos tectónicos; 3) las características neotectónicas; 4) la extensión geográfica; 5) la composición y configuración del relieve; 6) la sismicidad (histórica e instrumental); 7) los mecanismos focales; 8) la estructura litosférica. La estructura jerárquica considera que MX es una Región ST activa en las placas de Norteamérica y del Pacífico, diferenciada de otros territorios vecinos, incluso dentro de la primera placa. Ella está afectada por las placas de Caribe, Cocos y Pacífico, y la MPR, y todas participan en los procesos de interacción dinámica con AS, volcánica y tsunamis locales. En la Región ST se delimitan 3 PS (Figura 6) y en Cotilla et al. (1991) están los conceptos. Las 3 PS son: 1) Norte-Occidental (PC y Golfo de California); 2) Occidental (PV-Tehuantepec); 3) Centro-Oriental (Zonas del N y Centro-GM-Yucatán). La de mayor nivel es la segunda, donde están las zonas Colima, PV y OX. Cada PS tiene US, y en éstas están las zonas sismogenéticas; muchas de ellas con continuidad a regiones vecinas. Las 11 US (Figura 6) tienen varias características (Tabla 13). La prolongación de zonas activas a otras regiones se justifica con PEMEX (1982) cuando representa 48 Provincias Geológicas (23 Provincias terrestres, 8 Provincias marinas y 17 Provincias mixtas) en la figura 1, bajo el siguiente concepto:

[...]Provincia Geológica es aquella superficie cartografiable de la superficie sólida del Planeta, de centenares de kilómetros cuadrados de extensión, caracterizada por sus rocas, su estructura y una secuencia de eventos tal que integre una historia evolutiva singular diferente a la de sus áreas adyacentes, de las cuales están separadas por límites estratigráficos, tectónicos, o ambos [...]. 


\section{Figura 6. Provincias y Unidades sismotectónicas}

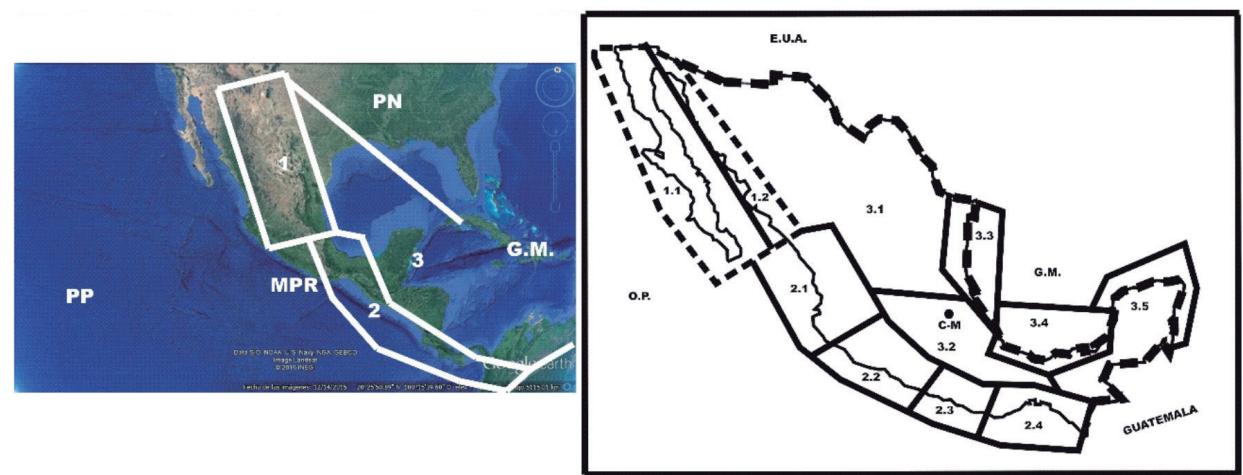

Provincias: 1) Placas $(\mathrm{PC}=$ Caribe, $\mathrm{PCO}=\mathrm{Cocos}, \mathrm{PN}=$ Norteamérica, $\mathrm{PNA}=$ Nazca, $\mathrm{PP}=\mathrm{Pacifi}-$ co, $\mathrm{PS}=$ Suramérica); 2) Microplaca (Rivera); 3) Provincias (áreas indicadas por líneas blancas $(1=$ Norte-Occidental, $2=$ Occidental, $3=$ Centro-Oriental $)$ ) 4$)$ Lugares $(\mathrm{AC}=$ América Central, $\mathrm{C}=$ Cuba, $\mathrm{F}=$ Florida).

Unidades: 1) Lugares (C-M= Ciudad de México, E.U.A.= Estados Unidos de América, G.M.= Golfo de México, O.P.= Océano Pacífico); 2) Unidades Sismotectónicas (1= Provincia Norte Occidental $(1.1=$ Península, $1.2=$ Golfo-Llanura Costera); $2=$ Provincia Occidental $(2.1=$ Nayarit-Puerto Vallarta, 2.2= Colima-Michoacán, $2.3=$ Guerrero, 2.4= Oaxaca-Chiapas); $3=$ Provincia CentroOriental (3.1=Interior, 3.2= México, 3.3= Norte de Veracruz, 3.4= Veracruz, 3.5= Yucatán)).

Tabla 13. Principales características de las Provincias Sismotectónicas

\begin{tabular}{|c|c|c|c|}
\hline Características & Norte-Occidental & Occidental & Centro-Oriental \\
\hline Identificación & 1 & 2 & 3 \\
Unidades & 2 & 4 & 5 \\
Mmáx & 7,2 & 8,2 & 8,0 \\
hmáx $(\mathrm{km})$ & 20 & 60 & $10-200$ \\
Terremotos / año & $\sim 1.000$ & $\sim 20.000$ & $\sim 600$ \\
Terremotos $(\mathrm{M}>7,5 />8,2)$ & $3 /-$ & $+50 /+6$ & $4 /-$ \\
Tipo de sismicidad & Entre placas & Entre placas & Interior de placas \\
Zona Sísmica & B-C-D & C-D & A-B \\
Estructura asociada & Fallas San Andrés-Cerro & Fosa Mesoamericana & Golfo de México- \\
Fuente de tsunamis & Prieto-Amado-Wagner & Oriente de México \\
Hmáx (m) de las olas & Lejana & Local y lejana & Lejana \\
\hline \multirow{2}{*}{ cantidad) Placas } & 2,5 & 10 & - \\
\hline Peligro & (2) Norteamérica y & (4) Caribe, Cocos, & (1) Norteamérica \\
& Pacífico & Norteamérica, Rivera & II-III \\
\hline
\end{tabular}


Las US delimitadas en la PS Norte-Occidental (contacto transcurrente) son 2: 1) Península (Bahía Tortugas-San Lucas-La Paz-Angostura), 2) Golfo-Llanura Costera (San Luis-Puerto Peñasco-La Yuta-Los MochisMazatlán). La PS Centro-Oriental tiene única y directa relación con la placa de Norteamérica, y es la de menor categoría; pero de mayor área, y tiene 5 US: 1) Interior, 2) México, 3) Norte de Veracruz, 4) Veracruz (VeracruzTabasco-Campeche) es la más activa, 5) Yucatán. Todas con AS de interior de placa, y mayoría de terremotos $(\mathrm{h}=20-25 \mathrm{~km})$. La PS Occidental tiene el mayor peligro de tsunamis, y los terremotos con $\mathrm{h}<50 \mathrm{~km}$ y mecanismos principalmente de subducción. Sus 4 US (Nayarit-PV, Colima-Michoacán, Guerrero, OX-Chiapas) tienen características comunes por la relación con la FM y las placas del Pacífico, en una región convergente.

Se ha visto que los estudios recogen datos sobre los peligros naturales, y a partir de ellos, y con la base cierta de los terremotos $(\mathrm{M}>7,0)$, tsunamis, volcanes y la cantidad de pérdidas humanas y económicas, se asumen 3 niveles de Peligro (I-III). Ellos por PS son: (I) Occidental, (II) Norte-Occidental y Centro-Oriental, y (III) Centro-Oriental.

Las estructuras de Guatemala y MX se relacionan con la interacción geodinámica (Cocos-Norteamérica y Cocos-Caribe). INSIVUMEH 2012 2014 muestra hay un mismo patrón sísmico y que: 1) las cantidades de terremotos locales/ año son similares (1.215 (2012), 1.734 (2013), 1.283 (2014)) (Tabla 14); 2) hay un arreglo lateral sucesivo y cuasi-paralelo de epicentros desde el OP al interior continental; 3) la AS disminuye en ese sentido. Este patrón está en los 3 pares de figuras 5 y 8 de sus boletines sismológicos. Las 3 figuras 8 tienen 6 zonas sismogenéticas, 5 de nuestro interés, de figura prácticamente rectangular. Las zonas G1-G4 son paralelas a la costa del OP y la G6 es transversal y tiene relación con el sistema Motagua-Polochic. Ésta posee la de menor cantidad de terremotos. Las G1-G4 se avienen, de forma aproximada a la dirección y características de la PS Occidental. Las proporciones de eventos en las 4 zonas (G1-G4)/ año (82\% (2014), $68 \%$ (2013), $70 \%$ (2012)) equivalen a las de GuerreroOX-Chiapas (85\% (2017), 75 \% (2016), 77 \% (2015), 78 \% (2012), 65 $\%$ (2011)). La cantidad es mayor en MX (22.503 (2017), 11.656 (2016), 8.472 (2015)) y como comprobación está la tabla 15. 
Tabla 14. Datos de la sismicidad de Guatemala (INSIVUMEH)

\begin{tabular}{|c|c|c|c|c|c|}
\hline Zona sismogenética Guatemala & Siglas & $\begin{array}{c}\text { Eventos } \\
2012\end{array}$ & $\begin{array}{c}\text { Eventos } \\
2013\end{array}$ & $\begin{array}{c}\text { Eventos } \\
2014\end{array}$ & Total \\
\hline Polochic Motagua Oeste & G6 & 14 & 180 & 152 & 346 \\
\hline Pacífico Central & G1 & 429 & 395 & 404 & 1.229 \\
\hline Antearco & $\mathrm{G} 2$ & 324 & 493 & 462 & 1.279 \\
\hline Arco Volcánico O-Arco Volcánico E & G3-G4 & 98 & 284 & 185 & 567 \\
\hline & $\Sigma G 1-G 4$ & 851 & 1.172 & 1.051 & 3.074 \\
\hline
\end{tabular}

Tabla 15. Datos de la sismicidad principal de México del año 2018 (enero-marzo)

\begin{tabular}{|c|c|c|c|}
\hline Mes & $\begin{array}{l}\text { Total de terremotos/ } \\
\text { Estado con mayor }(\%)\end{array}$ & M & $\begin{array}{l}\text { Mmáx/ h(km)/ Fecha/ Coordenada / Lugar/ } \\
\text { mecanismo/ réplicas }\end{array}$ \\
\hline Enero & 2.575/ Oaxaca (62) & $2,2-6,3$ & $\begin{array}{c}\text { 6,3/ 16/ 19.01/ 26,66 N 111,11 O/ Loreto/ } \\
\text { transcurrente/ } 22\end{array}$ \\
\hline Febrero & 4.165/ Oaxaca (60) & $1,5-7,2$ & $\begin{array}{c}\text { 7,2/ } 12 \text { / 16.02/ } 16,25 \mathrm{~N} 98,03 \mathrm{O} / \text { Oaxaca/ inverso/ } \\
4.894\end{array}$ \\
\hline Marzo & 3.178/ Oaxaca (71) & $1,6-5,3$ & $5,3 / 13 / 20.03 / 15,87$ N 98,72 O/ Oaxaca/ - / 60 \\
\hline
\end{tabular}

Lo expuesto demuestra que MX puede sufrir terremotos fuertes y tsunamis, con importantes pérdidas. Esto justifica la regionalización que servirá al MS. Se sostiene que MX es una región ST, mayoritariamente, de la placa de Norteamérica con 2 tipos de sismicidad (entreplacas y de interior de placa). Hay una estructura jerárquica con Provincias, Unidades y Zonas Sismogenéticas, y sus elementos sismotectónicos tienen continuidad en Estados Unidos de Norteamérica y Guatemala.

\section{Agradecimientos}

Al Centro de Sismología y Volcanología por los vehículos, combustible y coordinación del personal. Adán Gómez, Juan Pinzón y Leonardo Rivera fueron fundamentales en tareas de campo. Diana Núnez recopiló separtas y mapas. Muchas propietarios de fincas facilitaron la labor de campo. Autoridades locales y regionales ayudaron con profesionalidad. Salvador Crespillo por la preparación de los medios. Amador García preparó las figuras. La Hemeroteca (UCM) realizó las búsquedas bibliográficas. Al Departamento de Física de la Tierra y Astrofísica, por el uso de las 
Mario Octavio Cotilla-Rodríguez - Diego Córdoba-Barba - Francisco Javier Núnez-Cornú

Caracterización Sismotectónica de México

instalaciones. Los fondos son de: CGL2011-29474-C02-01 Plan Nacional I+D+i España; CONACYT-FOMIXJAL 2012-08-189965, México.

\section{Referencias}

Arce, M. F., Molina, E., Havskov, J. y Atakan, K. (1998) Tsunamis in Central America. Tech. Rep., 11:1-12. Reduction of Natural Disasters in Central America. Earthquake Preparedness and Hazard Mitigation. Inst. Solid Earth Physics, Univ. Bergen, Norway.

Arnaiz, M.S. y Garzón, Y. (2012). Nuevos mapas de anomalías gravimétricas del Caribe. Interciencia, 37(3), 176-182.

Authemayou, Ch., Brocard, G., Teyssier, Ch., Simon-Labric, T., Guttiérrez-Orrego, A., Cacao, E.N. y Morán, S. (2011). The CaribbeanNorth America-Cocos triple junction and the dynamics of the Polochic-Motagua fault system: Pull-up and zipper models. Tectonics, 30, doi:10.1029/2010TC002814.

Authemayou, Ch., Brocard, G., Teyssier, Ch., Suski, B., Cosenza, B., Morán-Ical, S., González, W., Véliz C., Aguilar-Hengstenberg, M.A., y Holliger, K. (2012). Quaternary seismo-tectonic activity of the Polochic fault, Guatemala. J. Geophys.Res., 117, B7403, doi:10.1029/2012JB009444.

Azuz, I. y Rivera, E. (2009). Descripción de la dinámica poblacional en la zona costera mexicana durante el periodo 2000-2005. Papeles de Población, 62, 75-107.

Benz, H.M., Dart, R.L., Villaseñor, A., Hayes, G.P., Tarr, A.C., Furlong, K.P. y Rhea, S. (2010) Seismicity of the Earth 1900-2010 Mexico and vicinity. U.S. Geological Survey Open-File Report 2010-1083-F.

Brink, U.T., Twichell, D., Lynett, P., Geist, E., Chaytor, J., Lee, H., Berczkowski, B. y Flores, C. (2009) Regional assessment of tsunamis potential in the Gulf of Mexico. Report to the National Tsunami Hazard Mitigation Program. USGS.

CENAPRED. (2001) Programa especial de prevención y mitigación del riesgo de desastres, 2001-2006. Secretaría de Gobernación. ISBN: 970-628-594-6, México, D.F.

Cotilla, M.O. (2017). Evaluation of recent intraplate seismic activity on Cochinos Bay, Cuba. Revista Geográfica de América Central, 58, 195-222. 
Cotilla, M.O., Bankwitz, P., Álvarez, L., Franzke, H.J., González, E., Grünthal, G., Pilarski, J., Díaz ,J. y Arteaga, F. (1991). Mapa sismotectónico de Cuba, escala 1:1.000.000. Comunicaciones Científicas sobre Geofisica y Astronomía, 3, 25 p.

Cotilla, M., Córdoba, D., Núñez, F., Gómez, A., Pinzón, J.I., y Rivera, L. (2017). Morfotectónica de Jalisco y Oaxaca (1), México. Revista Geográfica de América Central, 59, 197-248.

Dañobeitia, J.J., Bartolomé, R., Núñez, F., Córdoba, D., Bandy, W., Prada, M., Nuñez, D., Castellón, A. y Alonso, J.L. (2016). Crustal architecture at the collision zone between Rivera and North American plates at the Jalisco block: TSUJAL project. Pure appl.geophys., 173, 3.553-3.573.

DeMets, C., Mattioli, G., Jansma, P., Rogers, R., Tenori, C. y Tuner, H. (2007). Present motion and deformation of the Caribbean plate: Constraints from new GPS geodetic measurements from Honduras and Nicaragua. Bull. Seism. Soc. Am., 428, 21-36.

DeShon, H., Schwartz, S., Bilek, S., Dorman, T., González, V., Protti, J., Flueh, E. y Dixon, T. (2003). Sismogenic zone structure of the southern Middle America Trench, Costa Rica. J.Geophys.Res., 108, 2.491-2.294.

Ego, F. y Ansan, V.D. (2002). Why is the Central Trans Mexican Volcanic Belt $\left(102^{\circ}-99^{\circ} \mathrm{W}\right)$ in transtensive deformation. Tectonophysics, 329, 189-205.

Esteva, L. (1970). Regionalización sísmica de México para fines de ingeniería. Serie Azul, 246, 1-33.

Farreras, S. (1997). Tsunamis en México. Editor: M.F. Lavín. En: Contribuciones a la Oceanografía Física en México, Monografía No. 3. Unión Geofísica Mexicana, 75-98.

Franco, S.I., Canet, C., Iglesias, A. y Valdés, C. (2013). Seismic activity in the Gulf of Mexico: A preliminary analysis. Bol. Soc. Geol. Mexica$n a, 65,447-455$.

Frez, J. y González, J.J. (1991). Crustal structure and seismotectonics of northern Baja California. En: The Gulf and Peninsular Provinces of the California. Editores: J.P. Dauphin y B.T. Simoneit. American Association of Petroleum Geologists Memoir 47, 261-283. 
Geophysics Data Center / World Data Service (NGDC / WDS): Global historical tsunami database. doi:10.7289/V5TD9V7K, 2017. http:// www-worlddata.info/america/mexico/tsunamis.php

Guendel, F. y Protti, M. (1998). Sismicidad y sismotectónica de América Central. Fisica de la Tierra, 10, 19-51.

Gusiakov, V. (2002). Basic Pacific tsunami catalog and database, 47 BC2000 AD: Results of the first stage of the project. Tsunami Laboratory, Institute of Computational Mathematics and Mathematical Geophysics, Russian Academy of Sciences, Novosibirsk, Russia.

Guzman, M. y Gómez, J.M. (2006). Seismic strain rate along the Middle America Trench reveals significant differences between Cocos-North America and Cocos-Caribbean convergence. Geophysics. J. Int., $166,179-185$.

Guzman, M. y Meneses, J. J. (2000). The North America Caribbean plate boundary west of the Motagua-Polochic fault system: A fault jog in southeastern Mexico. Jour. of South Amer. Earth Sciences, 13, 459-468.

IG- Instituto de Geofísica (2010). Cien años de sismicidad en México. http://usuarios.geofisica.unam.mx/vladimir/sismos/100a\%F1os.html INEGI- Instituto Nacional de Estadísticas, Geografía e Informática (2005).

Provincias fisiográficas de México. Dirección General de Geografía. INEGI- Instituto Nacional de Estadísticas, Geografía e Informática. (2005 ) Zonas sísmicas y principales volcánicas de México. Dirección General de Geografía.

INEGI- Instituto Nacional de Estadísticas, Geografía e Informática (1981). Carta fisiográfica de la República Mexicana (8 hojas escala 1:1.000.000). Secretaría de Programación y Presupuesto, México.

INSIVUMEH- Instituto Nacional de Sismología, Vulcanología, Meteorología e Hidrología. Boletines anuales 2012, 2013, 2014, de Guatemala. http://www.insivumeh.gob.gt/

Kuroiwa, J. (1995) Tsunamis, evacuación de la población y planes de uso del suelo para mitigación de desastres. Localidades estudiadas entre 1981 y 1994. Editado por DHA, Ginebra y Lima.

Lanza, G. de la, Ortiz, M.A. y Carbajal, J.L. (2013). Diferenciación hidrogeomorfológica de los ambientes costeros del Pacífico, del Golfo de México y del Mar Caribe. Investigaciones Geográficas, 81, 33-50. 
Lockridge, P.A. y Smith, R.H. (1984). Tsunamis in the Pacific Basin: 19001983. US NOAA, National Geophysical Data Center.

Lugo, H.L. (1990). El relieve de la República Mexicana. Revista Inst. Geol., 9(1), 82-111.

Lyon, H., Barrier, E., Lasserre, C., Franco, A., Arzu, I., Chiquin, L., Chiquin, M., Duquesnoy, T., Flores, O., Galicia O., Luna, J., Molina, E., Porras, O., Requena, J., Robles, V., Romero, J. y Wolf, R. (2006). Kinematics of the North American-Caribbean-Cocos plates in Central America from new GPS measurements across the Polochic-Motagua fault system. Geophys.Res.Lett., 33, L19309, doi: 10.1029/2006GL027694.

Márquez, A., Cabral, E., Correa, F. y DeMets, Ch. (2004). A model for Mexican neotectonics based on nationwide GPS measurements, 1993-2001. Geofisica Internacional, 43(3), 319-330.

Medina, F. (1997). Sismicidady volcanes en México. ISBN 9789681668761. Michaud, F., Dañobeitia, J., Carbonell, R., Bartolomé, R., Córdoba, D., Delgado Argote, L., Núñez Cornú, F. y Monfret, T. (2000). New insights into the subducting ocean crust in the Middle American Trench off western Mexico (17 $\left.{ }^{\circ}-19^{\circ} \mathrm{N}\right)$. Tectonophysics, 318, 187-200.

Montero, P.W., Penaldo, H.G. y Rojas, Q.W. (1997). Informe final del Proyecto de Amenaza Sísmica de América Central. Universidad de Costa Rica, Instituto Panamericano de Geografía e Historia, Centro Internacional de Investigaciones para el Desarrollo, y Centro de Coordinación para la Prevención de Desastres Naturales de América Central.

Nava, A. (1997). Terremotos. Fondo de Cultura Económica. La Ciencia desde México, 34, 157,.

NOAA- National Oceanic and Atmospheric Administration. National Centers for Environmental Information, 2018. https://www.ngdc.noaaa. gov/hazard/tsu_db_shtml

Núñez, F. y Ponce, L. (1989). Zonas sísmicas de Oaxaca, México: Sismos máximos y tiempos de ocurrencia para el periodo 1542-1988. Geofisica Internacional, 28, 587-641.

Núñez, F. y Sánchez, C. (1999). Stress field estimations for Colima Volcano, Mexico, based in seismic data. Bull.Volcanol., 60, 568-580. 
Núñez, F.J., Cordoba, D., Dañobeitia, J.J. y TsuJal Team (2016). Geophysical studies across Rivera plate and Jalisco block, Mexico: TSUJAL Project. Seism. Res. Lett., 87(1), 59-72.

Núñez, F.J., Reyes, G.A., Rutz, M., Trejo, E., Carmena, M.A. y Ramírez, C.A. (2004). The 2003 Armería, México earthquake (Mw 7,4): Mainland and early aftershocks. Seism. Res. Lett., 75, 734-743.

Núñez, F.J., Reyes, G.A., Suárez, C., Nava, F.A., Rutz M., Ramírez, A. y Reyes A. (1998). Eventos sísmicos en el bloque Jalisco. GEOS, S-12, 286.

Núñez, J., Rutz, M., Nava, F.A., Reyes, G. y Suárez, C. (2002). Characteristics of seismicity in the coast north of Jalisco block, Mexico. Phys. Earth Plan. Int., 132, 141-144.

NUREG- 1451 (1992). Staff Technical Position on Investigations to identify fault displacements hazards and seismic hazards at a geologic repository. (McConnell, Blackford M.E. e Ibrahim A.K.), US Nuclear Regulatory Commission, Washington, D.C.

Pardo, M. y Suárez, G. (1995). Shape of the subducted Rivera and Cocos plates in southern Mexico: Seismic and tectonic implications. J.Geophys.Res., 100, 12.357-12.373.

Pavoni, N. (1985). Guidelines for the construction of seismotectonic maps. Tectonophysics, 117, 1-6.

PEMEX-Pemex Exploración y Producción (1982). Provincias geológicas de México.

Ramírez, Ma T. (2011). Evidencias ambientales de cambios de nivel de la costa del Pacífico de México: Terremotos y tsunamis. Revista de Geografia Norte Grande, 49, 99-124.

Ramos, L.G., Medina, H.C. y Montalvo, J.C. (2012). Patrones de sismicidad en la curvatura de Monterrey, NE de México. Revista Mexicana de Ciencias Geológicas, 29(3), 572-589.

Regulatory Guide 1.165. (1997). Identification and characterization of seismic sources and determination of safe shutdown earthquake ground motion.

RESIS II. (2008). Proyecto para la evaluación de la amenaza sísmica en Costa Rica.

Rutz, M. y Núñez, F.J. (2004). Sismotectónica del Norte y Oeste del Bloque de Jalisco usando datos sísmicos regionales. GEOS, 24(1), 2-13. 
Sánchez, A.J. y Farreras, S.F. (1993). Estudio de tsunamis en la costa occidental de México. World Data Center for solid Earth Geophysics, Publication SE-50, NOAA. Boulder, Colorado, USA.

SEMAR- Secretaría de Marina (2012). Historia de los tsunamis locales ocurridos en México. Centro de Alerta de Tsunamis.

SGM- Servicio Geológico Mexicano (2007). Atlas de Peligros del Estado de Chiapas. Informe Técnico del SG de México y la Secretaría de Seguridad Pública.

Singh, S.K., Pacheco, J.F., Pérez, X., Ordaz, M., y Reinoso, M. (2015). The 6 September $1997\left(M_{\mathrm{w}} 4.5\right)$ Coatzacoalcos-Minatitlán, Veracruz, Mexico earthquake: Implications for tectonics and seismic hazard of the region. Geofisica Internacional, 54(3), 289-298.

SPNM- Servicio Portuario Nacional de México (2017). https://www.sct. gob.mx/puertos-y-marina/puertos-de-mexico/.html

SSN- Servicio Sismológico Nacional UNAM. (2000). Catálogo cien años de sismicidad en México.

SSN- Servicio Sismológico de México (2017). Sismología de México.

https://www.sgm.gob.mx/Web/MuseoVirtual/Riesgos-geologicos/Sismologia-de-Mexico.html

Soloviev, S.L. y Go, Ch. (1975). A catalogue of tsunami on the eastern shore of the Pacific Ocean. Nauka Publishing House, Moscú.

Suárez, G. y Albini, P. (2009). Evidence for great tsunamigenic earthquakes (M 8.6) along the Mexican Subduction Zone. Bull.Seism.Soc.Am., 99(2A), 892-896.

Suarez, G. y Comte, D. (1992). Comparative analysis of seismogenic coupling in Chile and Mexico: The role of the upper lithospheric thickness. En: Wadati Conference on Great Subduction Earthquakes, September 16-17. Editores: D. Christensen et al., 65-66 pp. Geophys. Inst. Alaska Univ., Fairbanks.

Torres, M.A. (2010). Historical seismicity in Mexico during 1568-1837: Intensity evaluation and data reliability. Natural Hazards, 54(3), 863-878.

Trifonov, G.V. y Machette, N.M. (1993). The world map of major active faults project. Annali di Geofisica, 36 (3-4), 225-236.

UNAM- Servicio Sismológico Nacional de México. (2017). Datos de los sismos de México. México. 
Verma, S.P. (1985). Mexican volcanic belt. Boletín Geofísico Internacional, 24(1), 83-96.

Wheeler, R.L. y Krystinik, K.B. (1827). Segmentation of the Wasatch fault zone, Utah. Summaries, analyses, and interpretations of geological and geophysical data. US Geological Survey Bulletin.

WSM- World Stress (2016). Map Database Release. Editores: O. Heidbach, M. Rajabi, K. Reiter y M. Ziegler- WSM Team. The Helmholtz Centre Potsdam - GFZ German Research Centre for Geosciences, doi.org/10.5880/WSM.2016.001.

Zúñiga, R., Suárez, G., Ordaz, M. y García, V. (1997). Proyecto peligro sísmico en Norteamérica y el Caribe. Reporte Final. Capítulo 2: México. Centro Internacional e Investigaciones para el desarrollo. Otawa, Canada. Proyecto 89-0190. IPGH, 84 p.

Zúñiga, F.R., Suárez G., Figueroa, Soto A. y Mendoza, A. (2017). A firstorder seismotectonic regionalization of Mexico for seismic hazard and risk estimation. J. Seismology, 21(6), 1.295-1.322. 